\title{
Spatial distribution and galactic model parameters of cataclysmic variables
}

\author{
T. Ak ${ }^{a}{ }^{*}$, S. Bilir ${ }^{a}$, S. Ak ${ }^{a}$, Z. Eker ${ }^{b, c}$ \\ ${ }^{a}$ Istanbul University, Faculty of Sciences, Department of Astronomy and Space \\ Sciences, 34119 University, Istanbul, Turkey \\ ${ }^{\mathrm{b}}$ Canakkale Onsekiz Mart University, Faculty of Sciences and Arts, Ulupınar \\ Astrophysical Observatory, 17100, Çanakkale, Turkey \\ ${ }^{c}$ TÜBITAK National Observatory, Akdeniz University Campus, 07058 Antalya, \\ Turkey
}

\begin{abstract}
The spatial distribution, galactic model parameters and luminosity function of cataclysmic variables (CVs) in the solar neighbourhood have been determined from a carefully established sample of $459 \mathrm{CVs}$. The sample contains all of the CVs with distances computed from the Period-Luminosity-Colours (PLCs) relation of CVs which has been recently derived and calibrated with $2 M A S S$ photometric data. It has been found that an exponential function fits best to the observational $z$ distributions of all of the CVs in the sample, non-magnetic CVs and dwarf novae, while the $s e c h^{2}$ function is more appropriate for nova-like stars and polars. The vertical scaleheight of CVs is $158 \pm 14 \mathrm{pc}$ for the $2 M A S S J$-band limiting apparent magnitude of 15.8. On the other hand, the vertical scaleheights are $128 \pm 20$ and $160 \pm 5$ pc for dwarf novae and nova-like stars, respectively. The local space density of $\mathrm{CVs}$ is found to be $\sim 3 \times 10^{-5} p c^{-3}$ which is in agreement with the lower limit of the theoretical predictions. The luminosity function of CVs shows an increasing trend toward higher space densities at low luminosities, implying that the number of short-period systems should be high. The discrepancies between the theoretical and observational population studies of CVs will almost disappear if for the $z$ dependence of the space density the $\operatorname{sech}^{2}$ density function is used.
\end{abstract}

PACS: 97.80.Gm; 97.10Yp; 97.10.Xq; 98.35.Pr

Key words: Stars: Cataclysmic variables, Stars: statistics, Stars: Luminosity function, Galaxy: Solar neighbourhood

\footnotetext{
* corresponding author.

Email address: tanselak@istanbul.edu.tr (T. Ak).
} 


\section{Introduction}

Cataclysmic variables (hereafter $\mathrm{CVs}$ ) are short period semidetached interacting binary systems containing a white dwarf as a primary which accretes matter from a Roche-lobe-filled red dwarf companion (Knigge, 2006) typically via an accretion disc. A bright spot is formed in the location where the matter stream impacts on the accretion disc. In the CVs with strong magnetic primaries, the magnetic field is strong enough to prevent the formation of an accretion disc. Disc formation could be prevented, but accretion continues through channels as accretion flows. For a detailed description of the CV phenomenon and its subclasses see Warner (1995) and Hellier (2001).

Many basic properties of CVs, such as the masses of the component stars, cannot be directly determined (Smith \& Dhillon, 1998). The orbital period $P_{\text {orb }}$ is one of the exceptions as it is known directly from observations with a fair accuracy. Orbital periods with sufficient accuracy are available now for more than 640 systems (Downes et al., 2001; Ritter \& Kolb, 2003). Since an orbital period distribution is a useful indicator of dynamical evolution of orbits, most studies which are interested in the evolution of CVs are mainly concentrated on the distribution of orbital periods. The most striking features of the distribution of CV periods are the period gap and a sharp cut-off at about $80 \mathrm{~min}$ (Spruit \& Ritter, 1983; Hameury et al., 1988; Howell. Nelson \& Rappaport, 2001; Willems et al., 2005; Pretorius, Knigge \& Kolb, 2007).

Galactic space and velocity distributions could be crucial for various types of objects to indicate a general scenario or a stage on a scenario about their evolution. Any proposed evolutionary scheme must not be in conflict with data from stellar statistics (Duerbeck, 1984). If the space densities of systems in various intervals of orbital periods were known this would seriously improve our understanding of CV evolution. However, observational selection effects are usually strong. Therefore, even a rough estimate of space density may help to constrain the population models (Patterson, 1984). Standard models for the population of CVs predict a galactic space density of $10^{-5}-10^{-4} \mathrm{pc}^{-3}$ (Ritter \& Burkert, 1986; de Kool, 1992; Politano, 1996; Kolb, 2001; Willems et al., 2005, 2006). These predictions are inconsistent with the observed space densities of $\sim 10^{-6}-10^{-5} \mathrm{pc}^{-3}$ (Warner, 1974; Patterson, 1984, 1998; Ringwald, 1993; Schwope et al., 2002; Aungwerojwit et al., 2005; Araujo-Betancor et al., 2005; Grindlay et al., 2005).

From the observational point of view, the first step of deriving the space density of CVs is to collect the most truthfull CV distances in a selected sample. Only after reliable distances are available, the observed surface angular density distribution of CVs on the sky could be converted to a galactic spatial distribution. Previous space density estimates of CVs have been made from 
the samples which include CVs with distances according to various methods. The problems of those early estimations are mostly due to inadequate number of CVs with reliable distances. Unfortunately, there is no single technique, no "magic bullet", for distance finding to CVs (Patterson, 1998). However, a single method, which is able to provide reliable distances of all $\mathrm{CVs}$, would be crucial in computing the true space distribution and the space density of these objects. Such a method was recently suggested by Ak et al. (2007). The suggested method uses period-luminosity-colours (PCLs) relation of CVs calibrated by $2 M A S S$ photometric data and trigonometric parallaxes for a sufficient number of CVs.

Nevertheless, the interstellar reddening is one of the important dilemmas in a distance determination. The reddening corrections have been made so far by using short-wavelength observations, column densities, reddening maps, in general. However, the reddening coefficients in optical wavelengths are higher than in infrared wavelengths. The distance estimation technique proposed by Ak et al. (2007) is less affected by interstellar reddening since it is based on infrared colours from $2 M A S S$ observations.

The aim of this paper is to derive the spatial distribution, galactic model parameters and luminosity function of CVs in the solar neighbourhood using the distances estimated by the new method proposed by Ak et al. (2007), which could be applicable to all CVs with reliable periods and colours. Consequently, a data sample containing 459 CVs has been collected and analysed. Description of data, reddening problem, distances corrected for reddening, completeness of sample and galactic model parameters are discussed in section 2. After the analysis regarding to space distributions and luminosity functions in Section 3 , conclusions are summarized in Section 4.

\section{The Data}

Table 1 contains $2 M A S S$ photometric data, colour excesses and orbital periods of $\mathrm{CVs}$ which were collected for this study. In addition to the columns of name, type, orbital period, galactic coordinates $(l, b)$, colour excess $E(B-V)$, apperent brightness $(J)$ and infrared colours $(J-H)$ and $\left(H-K_{s}\right)$, which are the basic observatinal data, the last two colums (absolute magnitude, distance) are added as predictions from the PLCs relation of Ak et al. (2007).

A preliminary list of cataclysmic variables with orbital periods is collected from Downes et al.'s 1 (2001) and Ritter and Kolb' 22 (2003) catalogues.

\footnotetext{
1 http://archive.stsci.edu/prepds/cvcat/

2 http://www.mpa-garching.mpg.de/RKcat/
} 
Superhump periods of SU UMa type dwarf novae, for which the orbital periods are not known, are accepted as orbital periods. The bias introduced by using the superhump periods should be negligible since the superhump periods are only a few per cent longer than the orbital periods (Patterson, 2001).

The $J, H$ and $K_{s}$ magnitudes of the sample stars were taken from the PointSource Catalogue and Atlas (Cutri et al., 2003; Skrutskie et al., 2006) which is based on the 2MASS (Two Micron All Sky Survey) observations. The 2MASS photometric system comprises Johnson's $J(1.25 \mu \mathrm{m})$ and $H(1.65 \mu \mathrm{m})$ bands with the addition of $K_{s}(2.17 \mu \mathrm{m})$ band, which is bluer than Johnson's $K$-band. The limiting apparent magnitudes are 15.8, 15.1 and 14.3, for the three bands respectively (Skrutskie et al., 2006). The number of CVs having an orbital period and $2 M A S S$ photometric data is 541 . However, 82 of them were removed because they are beyond the limits of applicability of the PLCs relation, which will be discussed later. Consequently, the number of CVs selected for this study was reduced to 459 and all are recorded in Table 1.

\subsection{Intrinsic colours and colour excesses}

Intrinsic $(J-H)_{0}$ and $\left(H-K_{s}\right)_{0}$ colours and an orbital period are the basic parameters needed for the PLCs relation of Ak et al. (2007) to provide an absolute magnitude of a $\mathrm{CV}$, from which its distance can be computed. However, unlike the observed colours, which are directly available from the photometric observations, derivation of the intrinsic colours requires one more step namely using various colour excess values, if spectroscopic observations are not available to reveal them independently. For estimating the $(J-H)_{0}$ and $\left(H-K_{s}\right)_{0}$ colours, for this study the colour excess of $E(B-V)$ has been used.

Although the $E(B-V)$ values for some CVs are given in Bruch \& Engel (1994), we have preferred to use their first order predictions from the Schlegel, Finkbeiner \& Davis (1998) maps by using NASA Extragalactic Databas 3 in order to obtain a self-consistent data set. This is because those and other available colour excess values in the literature are unreliable since some of them are only assumed values, rather than a result of an investigation. Schlegel, Finkbeiner \& Davis (1998) maps, on the other hand, provide only the $E(B-V)$ values according to galactic coordinates, which are modeled for any direction from Sun to the edges of our Galaxy as a consequence of extinction by galactic dust. Those rough colour excess values according to galactic latitude $(b)$ and longitude $(l)$ towards the directions of stars are shown by $E_{\infty}(B-V)$, which symbolically means up to infinity but actually up to the edge of the Galaxy. Therefore, the $E_{\infty}(B-V)$ values have to be reduced according to the actual distance of each

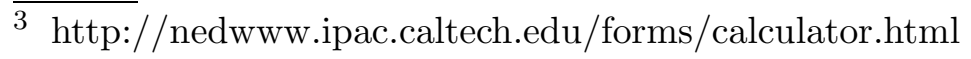


Table 1

The data sample. Types and orbital periods ( $P$, in days) were taken from Ritter and Kolb (2003). CV denotes CVs with unknown types, DN dwarf novae, NL nova-like stars, $\mathrm{N}$ novae and NR recurrent novae. $l$ and $b$ are galactic longitude and latitude in degrees, $J,(J-H)$ and $\left(H-K_{s}\right) 2 M A S S$ observations, $E(B-V)$ colour excess, $M_{J}$ the absolute magnitude in $J$-band and $d$ distance in pc.

\begin{tabular}{|c|c|c|c|c|c|c|c|c|c|c|}
\hline GCVS-Name & Type & $P$ & $l$ & $b$ & $E(B-V)$ & $J$ & $(J-H)$ & $\left(H-K_{s}\right)$ & $M(J)$ & $d$ \\
\hline $\mathrm{AM} \mathrm{CVn}$ & NL & 0.01191 & 140 & 79 & 0.013 & 14.482 & 0.039 & -0.203 & 8.69 & 143 \\
\hline HP Lib & NL & 0.01276 & 352 & 33 & 0.044 & 13.797 & -0.072 & 0.006 & 9.70 & 65 \\
\hline CR Boo & DN & 0.01703 & 341 & 66 & 0.022 & 14.548 & 0.012 & -0.295 & 7.03 & 316 \\
\hline V803 Cen & $\mathrm{DN}$ & 0.01866 & 309 & 21 & 0.037 & 13.806 & -0.029 & -0.023 & 8.67 & 105 \\
\hline J0926+3624 & NL & 0.01966 & 188 & 46 & 0.006 & 15.352 & 0.460 & 0.140 & 11.08 & 71 \\
\hline J1427-0123 & NL & 0.02539 & 346 & 53 & 0.011 & 13.975 & 0.560 & 0.268 & 11.64 & 29 \\
\hline GP Com & NL & 0.03234 & 324 & 80 & 0.013 & 15.724 & 0.104 & 0.475 & 11.38 & 73 \\
\hline EI Psc & $\mathrm{DN}$ & 0.04457 & 90 & -51 & 0.041 & 14.684 & 0.368 & 0.217 & 9.31 & 117 \\
\hline V396 Hya & NL & 0.04521 & 309 & 39 & 0.122 & 16.556 & 0.677 & -0.025 & 8.11 & 465 \\
\hline GW Lib & DN & 0.05332 & 341 & 27 & 0.095 & 16.191 & 0.605 & 0.193 & 9.18 & 243 \\
\hline $\mathrm{BW} \mathrm{Scl}$ & DN & 0.05432 & 345 & -74 & 0.007 & 15.835 & 0.342 & 0.555 & 11.32 & 80 \\
\hline DI UMa & DN & 0.05456 & 167 & 43 & 0.015 & 15.532 & 0.270 & 0.136 & 8.00 & 319 \\
\hline V844 Her & DN & 0.05464 & 62 & 44 & 0.007 & 16.763 & 0.479 & 0.206 & 9.08 & 343 \\
\hline $\mathrm{J} 0222+4122$ & $\mathrm{DN}$ & 0.0548 & 141 & -18 & 0.015 & 15.401 & 0.626 & 0.284 & 10.01 & 119 \\
\hline EV UMa & NL & 0.05534 & 118 & 63 & 0.015 & 16.317 & 0.308 & 0.289 & 9.20 & 264 \\
\hline GG Leo & NL & 0.05547 & 232 & 49 & 0.032 & 15.013 & 0.248 & 0.130 & 7.83 & 270 \\
\hline J0154-5947 & NL & 0.0556 & 289 & -56 & 0.011 & 15.991 & 0.330 & 0.512 & 10.90 & 104 \\
\hline J1238-0339 & $\mathrm{DN}$ & 0.0559 & 297 & 59 & 0.030 & 16.651 & 0.161 & 0.066 & 7.12 & 798 \\
\hline V386 Ser & $\mathrm{DN}$ & 0.05592 & 11 & 34 & 0.067 & 16.531 & 0.690 & 0.368 & 10.64 & 147 \\
\hline HV And & NL & 0.05599 & 121 & -19 & 0.072 & 15.372 & 0.226 & -0.016 & 6.58 & 556 \\
\hline CI Gru & $\mathrm{DN}$ & 0.056 & 359 & -47 & 0.020 & 16.138 & 0.333 & 0.294 & 9.26 & 236 \\
\hline $\mathrm{J} 0025+1217$ & $\mathrm{DN}$ & 0.0562 & 113 & -50 & 0.063 & 16.663 & 0.729 & 0.357 & 10.65 & 156 \\
\hline AL Com & $\mathrm{DN}$ & 0.05667 & 283 & 76 & 0.033 & 16.513 & 0.331 & 0.316 & 9.36 & 266 \\
\hline PU CMa & $\mathrm{DN}$ & 0.05669 & 234 & -13 & 0.026 & 12.486 & 0.025 & 0.072 & 6.77 & 137 \\
\hline WZ Sge & $\mathrm{DN}$ & 0.05669 & 58 & -8 & 0.023 & 14.862 & 0.305 & 0.559 & 11.11 & 56 \\
\hline SW UMa & $\mathrm{DN}$ & 0.05682 & 165 & 37 & 0.017 & 15.621 & 0.294 & 0.517 & 10.78 & 92 \\
\hline $\mathrm{J} 1839+2604$ & $\mathrm{DN}$ & 0.05689 & 55 & 14 & 0.011 & 13.399 & 0.546 & 0.311 & 9.92 & 49 \\
\hline $\mathrm{J} 1959+2242$ & $\mathrm{DN}$ & 0.058 & 61 & -4 & 0.186 & 16.562 & 0.832 & 0.161 & 9.12 & 285 \\
\hline RZ LMi & $\mathrm{DN}$ & 0.0585 & 191 & 51 & 0.014 & 15.679 & -0.015 & 0.056 & 6.50 & 681 \\
\hline $\mathrm{CC} \mathrm{Scl}$ & $\mathrm{DN}$ & 0.0587 & 17 & -69 & 0.013 & 16.040 & 0.191 & 0.445 & 9.91 & 168 \\
\hline V1025 Cen & NL & 0.05876 & 300 & 24 & 0.035 & 15.058 & 0.408 & 0.254 & 9.01 & 160 \\
\hline EG Cnc & $\mathrm{DN}$ & 0.05877 & 197 & 36 & 0.054 & 16.234 & 0.667 & -0.268 & 5.79 & 1203 \\
\hline T Leo (QZ Vir) & $\mathrm{DN}$ & 0.05882 & 263 & 60 & 0.007 & 14.771 & 0.436 & 0.509 & 11.02 & 56 \\
\hline RW UMi & $\mathrm{N}$ & 0.0591 & 110 & 33 & 0.036 & 16.338 & 0.463 & 0.199 & 8.73 & 327 \\
\hline FS Aur & $\mathrm{DN}$ & 0.0595 & 181 & 0 & 0.023 & 14.766 & 0.181 & 0.331 & 8.98 & 142 \\
\hline HT Cam & NL & 0.05971 & 153 & 31 & 0.030 & 16.000 & 0.024 & 0.388 & 8.97 & 252 \\
\hline DW Cnc & NL & 0.05979 & 205 & 22 & 0.012 & 14.654 & 0.318 & 0.303 & 9.14 & 126 \\
\hline $2219+1824$ & $\mathrm{DN}$ & 0.0599 & 80 & -32 & 0.017 & 15.976 & 0.536 & 0.561 & 11.60 & 75 \\
\hline V587 Lyr & $\mathrm{DN}$ & 0.06 & 70 & 9 & 0.067 & 15.144 & 0.554 & 0.073 & 7.93 & 270 \\
\hline V1040 Cen & DN & 0.0603 & 295 & 5 & 0.139 & 16.295 & 0.504 & 0.084 & 7.71 & 492 \\
\hline KX Aql & DN & 0.06036 & 50 & -2 & 0.092 & 15.844 & 1.001 & 0.197 & 9.93 & 147 \\
\hline FL Cet & NL & 0.06052 & 155 & -58 & 0.029 & 16.508 & 0.023 & 0.311 & 8.37 & 419 \\
\hline AQ Eri & DN & 0.06094 & 204 & -25 & 0.068 & 16.425 & 0.764 & 0.057 & 8.32 & 407 \\
\hline CP Pup & $\mathrm{N}$ & 0.06143 & 253 & -1 & 0.035 & 14.341 & 0.104 & 0.211 & 7.79 & 201 \\
\hline V4140 Sgr & $\mathrm{DN}$ & 0.06143 & 1 & -29 & 0.029 & 16.637 & -0.063 & 0.797 & 11.70 & 96 \\
\hline J1050-1404 & $\mathrm{DN}$ & 0.0615 & 264 & 39 & 0.037 & 16.740 & 0.544 & 0.460 & 10.76 & 155 \\
\hline J0918-2942 & $\mathrm{DN}$ & 0.06165 & 257 & 14 & 0.089 & 16.083 & 0.362 & 0.205 & 8.29 & 349 \\
\hline CP Tuc & NL & 0.06183 & 324 & -54 & 0.013 & 15.695 & 0.674 & 0.270 & 9.74 & 154 \\
\hline V1141 Aql & $\mathrm{DN}$ & 0.06202 & 41 & -9 & 0.092 & 16.931 & 1.123 & 0.226 & 10.40 & 195 \\
\hline V1159 Ori & $\mathrm{DN}$ & 0.06218 & 207 & -20 & 0.345 & 13.817 & 0.036 & 0.106 & 6.13 & 299 \\
\hline V2051 Oph & $\mathrm{DN}$ & 0.06243 & 358 & 8 & 0.056 & 14.327 & 0.455 & 0.342 & 9.58 & 87 \\
\hline V436 Cen & $\mathrm{DN}$ & 0.0625 & 282 & 21 & 0.033 & 14.220 & 0.362 & 0.332 & 9.31 & 94 \\
\hline V347 Pav & $\mathrm{NL}$ & 0.06255 & 320 & -26 & 0.037 & 16.201 & 0.679 & 0.537 & 11.64 & 81 \\
\hline EU UMa & NL & 0.0626 & 203 & 76 & 0.024 & 16.494 & 0.488 & 0.008 & 7.27 & 694 \\
\hline
\end{tabular}


Table 1 - continued

\begin{tabular}{|c|c|c|c|c|c|c|c|c|c|c|}
\hline GCVS-Name & Type & $P$ & $l$ & $b$ & $E(B-V)$ & $J$ & $(J-H)$ & $\left(H-K_{s}\right)$ & $M(J)$ & $d$ \\
\hline HO Del & $\mathrm{DN}$ & 0.0627 & 58 & -16 & 0.036 & 13.930 & 0.327 & 0.071 & 7.28 & 211 \\
\hline VY Aqr & $\mathrm{DN}$ & 0.06309 & 42 & -35 & 0.057 & 15.278 & 0.423 & 0.267 & 8.91 & 183 \\
\hline J1125-0016 & $\mathrm{DN}$ & 0.0631 & 263 & 56 & 0.010 & 12.395 & 0.694 & 0.134 & 8.74 & 54 \\
\hline OY Car & $\mathrm{DN}$ & 0.06312 & 290 & -11 & 0.029 & 14.953 & 0.518 & 0.338 & 9.75 & 108 \\
\hline J1600-4846 & $\mathrm{DN}$ & 0.06338 & 332 & 3 & 0.045 & 13.460 & 0.714 & 0.308 & 9.99 & 48 \\
\hline MR UMa & $\mathrm{DN}$ & 0.0636 & 163 & 67 & 0.012 & 13.399 & 0.644 & 0.133 & 8.58 & 91 \\
\hline ER UMa & $\mathrm{DN}$ & 0.06366 & 164 & 48 & 0.004 & 13.606 & 0.152 & -0.041 & 6.03 & 327 \\
\hline Var 79 Per & $\mathrm{DN}$ & 0.0637 & 87 & -14 & 0.083 & 14.759 & 0.579 & 0.150 & 8.38 & 183 \\
\hline DO Vul & $\mathrm{DN}$ & 0.064 & 57 & -4 & 0.071 & 15.602 & 0.313 & 0.315 & 8.92 & 211 \\
\hline EQ Cet & NL & 0.06446 & 193 & -81 & 0.007 & 16.727 & 0.404 & 0.579 & 11.23 & 125 \\
\hline UV Per & $\mathrm{DN}$ & 0.0649 & 133 & -4 & 0.098 & 16.468 & 0.742 & 0.295 & 9.79 & 208 \\
\hline $\mathrm{AK} \mathrm{Cnc}$ & $\mathrm{DN}$ & 0.0651 & 217 & 32 & 0.035 & 13.772 & 0.017 & 0.039 & 6.15 & 329 \\
\hline AO Oct & $\mathrm{DN}$ & 0.06535 & 318 & -34 & 0.067 & 15.503 & 0.481 & -0.131 & 6.02 & 767 \\
\hline J2050-0536 & NL & 0.06543 & 42 & -29 & 0.046 & 16.404 & 0.859 & 0.102 & 8.77 & 330 \\
\hline YY Sex & NL & 0.0656 & 253 & 45 & 0.044 & 16.476 & 0.928 & 0.157 & 9.35 & 262 \\
\hline V551 Sgr & $\mathrm{DN}$ & 0.0659 & 357 & -6 & 0.060 & 14.319 & 0.681 & 0.120 & 8.39 & 150 \\
\hline IX Dra & $\mathrm{DN}$ & 0.06646 & 97 & 29 & 0.034 & 16.470 & 0.181 & 0.286 & 8.35 & 415 \\
\hline $\mathrm{J} 0209+2832$ & NL & 0.06686 & 143 & -31 & 0.072 & 16.052 & 0.343 & -0.048 & 6.21 & 903 \\
\hline V550 Cyg & DN & 0.0672 & 70 & 0 & 0.093 & 14.592 & 0.426 & 0.182 & 8.06 & 195 \\
\hline SX LMi & DN & 0.0672 & 199 & 64 & 0.030 & 15.707 & 0.149 & 0.166 & 7.36 & 462 \\
\hline SS UMi & DN & 0.06778 & 106 & 39 & 0.037 & 15.874 & 0.355 & -0.248 & 4.80 & 1614 \\
\hline BZ UMa & DN & 0.06799 & 159 & 39 & 0.025 & 14.824 & 0.384 & 0.435 & 9.94 & 94 \\
\hline J0131-0901 & $\mathrm{CV}$ & 0.068 & 153 & -70 & 0.034 & 15.679 & 0.207 & -0.059 & 5.82 & 926 \\
\hline KS UMa & $\mathrm{DN}$ & 0.068 & 160 & 52 & 0.006 & 16.087 & 0.447 & 0.573 & 11.16 & 96 \\
\hline HS Cam & NL & 0.06821 & 150 & 27 & 0.051 & 16.726 & 0.755 & -0.001 & 7.62 & 648 \\
\hline EX Hya & NL & 0.06823 & 303 & 33 & 0.020 & 12.274 & 0.324 & 0.263 & 8.51 & 56 \\
\hline RZ Sge & $\mathrm{DN}$ & 0.06828 & 56 & -7 & 0.046 & 15.734 & 0.420 & 0.400 & 9.72 & 157 \\
\hline TY Psc & $\mathrm{DN}$ & 0.06833 & 131 & -30 & 0.053 & 13.226 & 0.081 & 0.045 & 6.20 & 249 \\
\hline IR Gem & $\mathrm{DN}$ & 0.0684 & 187 & 11 & 0.029 & 15.218 & 0.343 & 0.343 & 9.13 & 163 \\
\hline V699 Oph & $\mathrm{DN}$ & 0.0685 & 10 & 29 & 0.096 & 14.176 & 0.606 & 0.220 & 8.75 & 117 \\
\hline V393 Pav & NL & 0.06863 & 340 & -31 & 0.038 & 16.389 & 0.713 & 0.186 & 8.91 & 309 \\
\hline $\mathrm{J} 2303+0106$ & $\mathrm{DN}$ & 0.069 & 76 & -52 & 0.034 & 16.059 & 0.408 & 0.469 & 10.20 & 147 \\
\hline V1504 Cyg & $\mathrm{DN}$ & 0.06957 & 76 & 11 & 0.035 & 16.110 & 0.198 & 0.510 & 9.93 & 169 \\
\hline CY UMa & DN & 0.06957 & 159 & 59 & 0.009 & 16.012 & 0.512 & 0.469 & 10.50 & 126 \\
\hline VV Pup & NL & 0.06975 & 240 & 9 & 0.018 & 15.553 & 0.640 & 0.369 & 10.07 & 124 \\
\hline J1928-5001 & NL & 0.07016 & 348 & -26 & 0.030 & 13.411 & 0.467 & 0.151 & 7.97 & 121 \\
\hline BB Ari & $\mathrm{DN}$ & 0.0703 & 152 & -29 & 0.090 & 16.551 & 0.634 & 0.234 & 8.88 & 330 \\
\hline V834 Cen & NL & 0.0705 & 317 & 16 & 0.015 & 13.467 & 0.311 & 0.391 & 9.35 & 66 \\
\hline $\mathrm{J} 0803+2516$ & $\mathrm{DN}$ & 0.071 & 197 & 26 & 0.021 & 14.721 & 0.444 & 0.193 & 8.21 & 199 \\
\hline PU Per & $\mathrm{DN}$ & 0.0713 & 147 & -22 & 0.056 & 15.799 & 0.410 & 0.059 & 7.05 & 550 \\
\hline GD 552 & $\mathrm{DN}$ & 0.07134 & 110 & 4 & 0.098 & 15.385 & 0.587 & 0.340 & 9.48 & 145 \\
\hline FO And & $\mathrm{DN}$ & 0.07161 & 128 & -25 & 0.020 & 15.493 & -0.153 & 0.652 & 10.03 & 123 \\
\hline J0953+1458 & NL & 0.07205 & 220 & 47 & 0.031 & 16.911 & 0.530 & 0.289 & 9.08 & 364 \\
\hline AW Sge & DN & 0.0724 & 56 & -7 & 0.078 & 15.831 & 0.778 & 0.113 & 8.32 & 308 \\
\hline DT Oct & $\mathrm{DN}$ & 0.0726 & 310 & -27 & 0.111 & 14.442 & 0.236 & 0.167 & 7.23 & 265 \\
\hline EP Dra & NL & 0.07266 & 100 & 24 & 0.061 & 16.274 & 0.582 & 0.196 & 8.45 & 359 \\
\hline VZ Pyx & $\mathrm{DN}$ & 0.07332 & 250 & 14 & 0.048 & 14.186 & 0.315 & 0.259 & 8.22 & 153 \\
\hline $\mathrm{CC} \mathrm{Cnc}$ & $\mathrm{DN}$ & 0.07352 & 204 & 32 & 0.027 & 16.517 & 0.435 & 0.458 & 10.04 & 195 \\
\hline HT Cas & $\mathrm{DN}$ & 0.07365 & 125 & -2 & 0.029 & 14.703 & 0.477 & 0.383 & 9.59 & 104 \\
\hline AY Lyr & $\mathrm{DN}$ & 0.0737 & 67 & 18 & 0.029 & 16.795 & 0.531 & 0.464 & 10.33 & 194 \\
\hline V1251 Cyg & $\mathrm{DN}$ & 0.07376 & 94 & -3 & 0.222 & 15.626 & 0.136 & 0.133 & 6.43 & 630 \\
\hline IY UMa & $\mathrm{DN}$ & 0.07391 & 150 & 52 & 0.008 & 15.725 & 0.625 & 0.235 & 8.92 & 228 \\
\hline J1556-0009 & $\mathrm{DN}$ & 0.07408 & 9 & 38 & 0.072 & 16.285 & 0.544 & 0.475 & 10.34 & 150 \\
\hline VW Hyi & DN & 0.07427 & 285 & -38 & 0.025 & 12.522 & 0.485 & 0.335 & 9.24 & 45 \\
\hline Z Cha & $\mathrm{DN}$ & 0.0745 & 289 & -22 & 0.047 & 13.968 & 0.404 & 0.250 & 8.35 & 130 \\
\hline QW Ser & DN & 0.07457 & 13 & 49 & 0.024 & 16.274 & 0.325 & 0.557 & 10.46 & 144 \\
\hline J0242-2802 & $\mathrm{DN}$ & 0.0746 & 222 & -65 & 0.012 & 16.697 & 0.682 & 0.491 & 10.93 & 142 \\
\hline LY Hya & DN & 0.0748 & 313 & 32 & 0.031 & 16.137 & 0.463 & 0.466 & 10.12 & 158 \\
\hline WX Hyi & $\mathrm{DN}$ & 0.07481 & 289 & -51 & 0.019 & 13.482 & 0.244 & 0.277 & 8.19 & 114 \\
\hline
\end{tabular}


Table 1 - continued

\begin{tabular}{|c|c|c|c|c|c|c|c|c|c|c|}
\hline GCVS-Name & Type & $P$ & $l$ & $b$ & $E(B-V)$ & $J$ & $(J-H)$ & $\left(H-K_{s}\right)$ & $M(J)$ & $d$ \\
\hline BK Lyn & $\mathrm{NL}$ & 0.07498 & 191 & 45 & 0.015 & 14.482 & 0.020 & 0.099 & 6.29 & 432 \\
\hline FT Cam & $\mathrm{DN}$ & 0.07499 & 140 & 3 & 0.096 & 15.751 & 0.198 & 0.579 & 10.12 & 128 \\
\hline CE Gru & NL & 0.0754 & 357 & -48 & 0.018 & 16.356 & 0.456 & 0.205 & 8.19 & 427 \\
\hline V893 Sco & $\mathrm{DN}$ & 0.07596 & 348 & 16 & 0.093 & 13.222 & 0.314 & 0.226 & 7.79 & 117 \\
\hline WY Tri & $\mathrm{DN}$ & 0.07597 & 145 & -26 & 0.068 & 15.820 & 0.192 & 0.228 & 7.55 & 439 \\
\hline RZ Leo & $\mathrm{DN}$ & 0.07604 & 265 & 59 & 0.024 & 16.338 & 0.674 & 0.277 & 9.25 & 259 \\
\hline QY Per & $\mathrm{DN}$ & 0.0761 & 149 & -13 & 0.119 & 15.347 & -0.078 & 0.164 & 6.25 & 628 \\
\hline T Pyx & NR & 0.07622 & 257 & -1 & 0.088 & 15.149 & 0.187 & 0.278 & 7.85 & 278 \\
\hline $0417+7445$ & $\mathrm{DN}$ & 0.07632 & 136 & 17 & 0.051 & 16.014 & 0.599 & 0.418 & 10.03 & 154 \\
\hline SU UMa & DN & 0.07635 & 154 & 33 & 0.027 & 11.777 & 0.046 & 0.061 & 6.01 & 141 \\
\hline V630 Cyg & $\mathrm{DN}$ & 0.0764 & 88 & -8 & 0.150 & 14.679 & 0.176 & 0.102 & 6.38 & 429 \\
\hline $\mathrm{J} 1730+6247$ & DN & 0.07653 & 92 & 33 & 0.028 & 15.284 & 0.095 & -0.028 & 5.47 & 907 \\
\hline HS Vir & DN & 0.0769 & 324 & 52 & 0.037 & 15.016 & 0.146 & 0.267 & 7.75 & 280 \\
\hline CD Ind & NL & 0.07701 & 337 & -41 & 0.018 & 13.821 & 0.590 & 0.266 & 8.93 & 94 \\
\hline DH Aql & DN & 0.07738 & 28 & -12 & 0.154 & 15.932 & 0.669 & 0.039 & 7.15 & 535 \\
\hline V503 Cyg & DN & 0.0777 & 82 & 3 & 0.539 & 16.370 & 1.083 & 0.087 & 7.73 & 430 \\
\hline TY Vul & DN & 0.0777 & 69 & -10 & 0.134 & 15.602 & 0.168 & 0.117 & 6.47 & 636 \\
\hline PV Per & DN & 0.0778 & 146 & -20 & 0.040 & 15.181 & 0.036 & 0.193 & 6.88 & 449 \\
\hline V660 Her & DN & 0.07826 & 48 & 25 & 0.097 & 14.386 & -0.009 & -0.053 & 4.81 & 791 \\
\hline BZ Cir & DN & 0.0784 & 314 & -8 & 0.169 & 15.612 & 0.540 & -0.089 & 5.81 & 852 \\
\hline J0219-3045 & DN & 0.0784 & 229 & -70 & 0.019 & 16.391 & 0.720 & 0.312 & 9.56 & 231 \\
\hline V884 Her & NL & 0.07848 & 44 & 19 & 0.030 & 13.785 & 0.497 & 0.270 & 8.64 & 105 \\
\hline CU Vel & DN & 0.0785 & 264 & 3 & 0.152 & 14.492 & 0.503 & 0.146 & 7.48 & 237 \\
\hline MR Ser & NL & 0.0788 & 32 & 48 & 0.022 & 14.082 & 0.299 & 0.429 & 9.31 & 89 \\
\hline BL Hyi & NL & 0.07892 & 296 & -49 & 0.009 & 14.318 & 0.653 & 0.411 & 10.12 & 69 \\
\hline $\mathrm{J} 2100+0044$ & DN & 0.079 & 50 & -26 & 0.087 & 16.100 & -0.311 & 0.504 & 8.13 & 378 \\
\hline ST LMi & NL & 0.07909 & 212 & 66 & 0.009 & 13.510 & 0.416 & 0.187 & 7.85 & 135 \\
\hline RS Car & $\mathrm{N}$ & 0.0795 & 291 & -1 & 0.015 & 15.865 & 1.261 & 0.460 & 12.03 & 58 \\
\hline BR Lup & $\mathrm{DN}$ & 0.0795 & 334 & 12 & 0.203 & 15.179 & 0.042 & 0.059 & 5.50 & 794 \\
\hline NSV 9923 & DN & 0.0796 & 350 & -9 & 0.103 & 16.092 & 0.243 & 0.160 & 6.98 & 637 \\
\hline AN UMa & NL & 0.07975 & 166 & 62 & 0.009 & 15.601 & 0.126 & 0.407 & 8.70 & 239 \\
\hline WW Hor & NL & 0.0802 & 272 & -58 & 0.017 & 16.003 & 0.472 & 0.645 & 11.33 & 86 \\
\hline J0549-4921 & DN & 0.08022 & 256 & -30 & 0.044 & 15.619 & 0.409 & 0.341 & 8.86 & 221 \\
\hline AR UMa & NL & 0.0805 & 167 & 65 & 0.011 & 14.148 & 0.547 & 0.335 & 9.23 & 96 \\
\hline V1974 Cyg & $\mathrm{N}$ & 0.08126 & 89 & 8 & 0.191 & 15.672 & 0.389 & 0.078 & 6.51 & 628 \\
\hline TU Crt & DN & 0.08209 & 272 & 35 & 0.037 & 16.225 & 0.708 & 0.305 & 9.33 & 236 \\
\hline HV Aur & $\mathrm{DN}$ & 0.0823 & 166 & -4 & 0.132 & 13.665 & 0.210 & 0.140 & 6.60 & 245 \\
\hline QZ Ser & DN & 0.08316 & 35 & 47 & 0.036 & 15.652 & 0.578 & 0.391 & 9.59 & 161 \\
\hline V1007 Her & NL & 0.08328 & 66 & 33 & 0.023 & 14.032 & 0.302 & 0.117 & 6.88 & 267 \\
\hline TY PsA & DN & 0.0841 & 26 & -63 & 0.015 & 14.290 & 0.421 & 0.286 & 8.43 & 148 \\
\hline BF Ara & DN & 0.0845 & 344 & -8 & 0.169 & 14.788 & 0.178 & -0.013 & 5.24 & 758 \\
\hline KK Tel & $\mathrm{DN}$ & 0.0845 & 346 & -36 & 0.020 & 12.350 & 0.361 & 0.110 & 6.95 & 119 \\
\hline V452 Cas & DN & 0.0846 & 123 & -9 & 0.118 & 15.908 & 0.305 & 0.304 & 8.02 & 361 \\
\hline $\mathrm{J} 1629+2635$ & NL & 0.0847 & 45 & 42 & 0.043 & 15.458 & 0.625 & 0.163 & 7.97 & 309 \\
\hline V364 Peg & DN & 0.085 & 62 & -24 & 0.051 & 13.211 & 0.322 & 0.074 & 6.51 & 215 \\
\hline $\mathrm{J} 2234+0041$ & DN & 0.085 & 68 & -47 & 0.064 & 16.449 & 0.425 & 0.453 & 9.54 & 235 \\
\hline DV UMa & DN & 0.08585 & 175 & 49 & 0.011 & 16.894 & 1.026 & 0.072 & 8.38 & 502 \\
\hline V419 Lyr & $\mathrm{DN}$ & 0.0864 & 61 & 9 & 0.106 & 15.600 & 0.347 & 0.164 & 7.07 & 486 \\
\hline YZ Cnc & DN & 0.0868 & 194 & 28 & 0.025 & 13.166 & 0.215 & 0.122 & 6.59 & 205 \\
\hline $\mathrm{HU}$ Aqr & NL & 0.08682 & 45 & -33 & 0.063 & 14.177 & 0.284 & 0.259 & 7.69 & 194 \\
\hline IR Com & DN & 0.08704 & 278 & 83 & 0.031 & 15.032 & 0.421 & 0.029 & 6.41 & 524 \\
\hline EU Cnc & NL & 0.0871 & 216 & 32 & 0.018 & 13.328 & 0.464 & 0.042 & 6.65 & 215 \\
\hline J0738+2855 & $\mathrm{CV}$ & 0.0875 & 191 & 22 & 0.024 & 16.564 & 0.633 & 0.438 & 9.99 & 205 \\
\hline V344 Lyr & $\mathrm{DN}$ & 0.0876 & 73 & 19 & 0.046 & 15.605 & 0.173 & 0.149 & 6.61 & 619 \\
\hline UZ For & NL & 0.08787 & 220 & -53 & 0.012 & 15.317 & 0.420 & 0.121 & 7.11 & 437 \\
\hline J0924+0801 & NL & 0.088 & 224 & 38 & 0.041 & 16.252 & 0.419 & 0.397 & 9.08 & 268 \\
\hline GZ Cnc & DN & 0.0883 & 222 & 36 & 0.038 & 14.211 & 0.108 & 0.122 & 6.23 & 388 \\
\hline J1300-3052 & DN & 0.08898 & 305 & 32 & 0.072 & 16.465 & 0.480 & 0.288 & 8.33 & 411 \\
\hline GX Cas & DN & 0.089 & 123 & -5 & 0.107 & 16.226 & 0.688 & 0.193 & 8.09 & 405 \\
\hline
\end{tabular}


Table 1 - continued

\begin{tabular}{|c|c|c|c|c|c|c|c|c|c|c|}
\hline GCVS-Name & Type & $P$ & $l$ & $b$ & $E(B-V)$ & $J$ & $(J-H)$ & $\left(H-K_{s}\right)$ & $M(J)$ & $d$ \\
\hline UV Gem & $\mathrm{DN}$ & 0.089 & 195 & 6 & 0.208 & 16.520 & 0.235 & 0.295 & 7.45 & 598 \\
\hline $\mathrm{J} 1556+3523$ & $\mathrm{CV}$ & 0.0892 & 57 & 50 & 0.015 & 12.505 & 0.411 & 0.093 & 6.83 & 136 \\
\hline MT Dra & NL & 0.08939 & 85 & 23 & 0.040 & 16.086 & 0.444 & 0.300 & 8.39 & 341 \\
\hline UW Pic & NL & 0.09264 & 253 & -33 & 0.029 & 15.625 & 0.337 & 0.401 & 8.79 & 230 \\
\hline V381 Vel & NL & 0.0931 & 274 & 13 & 0.137 & 16.619 & 0.437 & 0.074 & 6.39 & 1052 \\
\hline V725 Aql & $\mathrm{DN}$ & 0.0944 & 50 & -9 & 0.043 & 11.532 & 0.329 & 0.037 & 6.00 & 125 \\
\hline V516 Pup & NL & 0.0952 & 263 & -9 & 0.063 & 16.255 & 0.307 & 0.638 & 10.32 & 150 \\
\hline QS Tel & NL & 0.09719 & 352 & -27 & 0.031 & 14.293 & 0.524 & 0.244 & 7.99 & 180 \\
\hline DD Cir & $\mathrm{N}$ & 0.09746 & 311 & -8 & 0.062 & 15.244 & 0.291 & 0.578 & 9.78 & 121 \\
\hline $\mathrm{J} 1702+3229$ & $\mathrm{DN}$ & 0.10008 & 55 & 36 & 0.021 & 15.632 & 0.582 & 0.236 & 8.03 & 328 \\
\hline V348 Pup & NL & 0.10184 & 248 & -12 & 0.155 & 14.865 & 0.125 & 0.180 & 6.10 & 532 \\
\hline IM Nor & NR & 0.1026 & 327 & 2 & 0.067 & 15.457 & 1.833 & 0.226 & 11.05 & 74 \\
\hline MN Dra & $\mathrm{DN}$ & 0.10424 & 99 & 15 & 0.254 & 16.140 & 0.358 & 0.349 & 7.68 & 444 \\
\hline $\mathrm{AP} \mathrm{CrB}$ & NL & 0.10546 & 44 & 50 & 0.027 & 14.714 & 0.416 & 0.410 & 8.74 & 155 \\
\hline V Per & $\mathrm{N}$ & 0.10713 & 133 & -5 & 0.182 & 15.950 & 0.393 & 0.095 & 5.98 & 915 \\
\hline V795 Her & NL & 0.10825 & 57 & 34 & 0.025 & 12.854 & -0.019 & 0.076 & 5.08 & 354 \\
\hline J0524+4244 & NL & 0.10915 & 166 & 4 & 0.450 & 16.289 & 0.556 & -0.076 & 4.51 & 1887 \\
\hline $\mathrm{J} 0813+2813$ & NL & 0.11 & 194 & 29 & 0.031 & 16.272 & 0.150 & 0.121 & 5.80 & 1226 \\
\hline V349 Pav & NL & 0.1109 & 331 & -33 & 0.032 & 16.112 & -0.117 & 0.768 & 9.86 & 175 \\
\hline QU Vul & $\mathrm{N}$ & 0.11177 & 69 & -6 & 0.052 & 12.504 & 0.693 & 0.164 & 7.44 & 101 \\
\hline V405 Vul & DN & 0.112 & 59 & -3 & 0.108 & 14.531 & 0.452 & 0.196 & 6.93 & 317 \\
\hline $\mathrm{J} 1803+4012$ & NL & 0.1126 & 67 & 26 & 0.023 & 15.237 & 0.477 & 0.363 & 8.40 & 231 \\
\hline J0752+3628 & NL & 0.114 & 184 & 27 & 0.056 & 15.224 & 0.597 & -0.365 & 3.24 & 2439 \\
\hline V592 Cas & NL & 0.11506 & 119 & -6 & 0.139 & 12.294 & 0.038 & 0.067 & 4.77 & 302 \\
\hline WX LMi & NL & 0.11592 & 183 & 58 & 0.006 & 13.427 & 0.594 & 0.340 & 8.50 & 96 \\
\hline $\mathrm{J} 1801-2722$ & $\mathrm{DN}$ & 0.117 & 3 & -2 & 0.083 & 14.024 & 0.935 & 0.221 & 8.32 & 134 \\
\hline TU Men & $\mathrm{DN}$ & 0.1172 & 289 & -33 & 0.064 & 14.747 & 0.584 & 0.315 & 8.13 & 205 \\
\hline V2214 Oph & $\mathrm{N}$ & 0.11752 & 355 & 6 & 0.164 & 15.592 & 0.663 & 0.319 & 8.14 & 289 \\
\hline V630 Sgr & $\mathrm{N}$ & 0.118 & 358 & -7 & 0.101 & 14.637 & 0.657 & 0.123 & 6.81 & 352 \\
\hline V351 Pup & $\mathrm{N}$ & 0.1182 & 253 & -1 & 0.086 & 16.526 & 0.547 & 0.298 & 7.84 & 527 \\
\hline V478 Her & $\mathrm{DN}$ & 0.12 & 46 & 30 & 0.069 & 16.047 & 0.492 & 0.205 & 7.01 & 623 \\
\hline V1084 Her & NL & 0.12056 & 56 & 40 & 0.017 & 12.459 & 0.041 & 0.080 & 5.02 & 305 \\
\hline DM Gem & $\mathrm{N}$ & 0.1228 & 185 & 12 & 0.094 & 14.479 & 0.276 & 0.041 & 5.13 & 712 \\
\hline V442 Oph & NL & 0.12433 & 9 & 9 & 0.197 & 13.329 & 0.100 & 0.113 & 4.95 & 437 \\
\hline LQ Peg & NL & 0.12475 & 66 & -29 & 0.095 & 14.383 & -0.066 & 0.116 & 4.76 & 809 \\
\hline V4633 Sgr & $\mathrm{N}$ & 0.12558 & 5 & -6 & 0.006 & 10.556 & -0.025 & 0.723 & 9.52 & 16 \\
\hline J0230-6842 & NL & 0.12625 & 290 & -46 & 0.027 & 16.807 & 1.115 & -0.131 & 6.12 & 1358 \\
\hline BX Pup & DN & 0.127 & 242 & 2 & 0.035 & 14.549 & 0.502 & 0.204 & 6.97 & 323 \\
\hline AH Men & NL & 0.12721 & 294 & -28 & 0.089 & 12.478 & 0.406 & 0.178 & 6.40 & 158 \\
\hline DN Gem & $\mathrm{N}$ & 0.12785 & 184 & 15 & 0.116 & 15.432 & 0.197 & -0.029 & 4.26 & 1634 \\
\hline KQ Mon & NL & 0.128 & 227 & 4 & 0.059 & 12.878 & 0.497 & 0.033 & 5.62 & 276 \\
\hline AM Her & NL & 0.12893 & 78 & 26 & 0.017 & 11.703 & 0.508 & 0.193 & 6.90 & 91 \\
\hline V1033 Cen & NL & 0.13152 & 295 & -2 & 0.445 & 13.531 & 0.381 & 0.213 & 5.74 & 302 \\
\hline $\mathrm{J} 1610+0352$ & NL & 0.13232 & 16 & 37 & 0.057 & 14.445 & 0.401 & 0.246 & 6.87 & 320 \\
\hline MV Lyr & NL & 0.13234 & 75 & 15 & 0.091 & 15.865 & 0.516 & 0.164 & 6.48 & 725 \\
\hline $\mathrm{J} 0837+3830$ & NL & 0.1325 & 184 & 37 & 0.038 & 16.562 & 0.620 & 0.375 & 8.42 & 418 \\
\hline J0809+3814 & NL & 0.133 & 183 & 31 & 0.048 & 15.288 & 0.089 & 0.319 & 6.60 & 537 \\
\hline $0728+6738$ & NL & 0.13362 & 148 & 29 & 0.034 & 15.138 & 0.330 & 0.285 & 7.00 & 419 \\
\hline V1494 Aql & $\mathrm{N}$ & 0.13461 & 41 & -5 & 0.192 & 14.848 & 0.479 & 0.239 & 6.68 & 398 \\
\hline $\mathrm{BG} \mathrm{CMi}$ & NL & 0.13475 & 208 & 13 & 0.032 & 14.550 & 0.268 & 0.143 & 5.77 & 564 \\
\hline SW Sex & NL & 0.13494 & 246 & 42 & 0.029 & 14.212 & 0.220 & 0.103 & 5.35 & 584 \\
\hline V393 Hya & NL & 0.135 & 276 & 27 & 0.066 & 15.591 & 0.188 & -0.088 & 3.78 & 2244 \\
\hline HL Aqr & NL & 0.1356 & 66 & 43 & 0.008 & 13.389 & 0.076 & 0.169 & 5.49 & 378 \\
\hline OR And & NL & 0.1359 & 106 & -10 & 0.179 & 14.167 & 0.031 & 0.108 & 4.55 & 780 \\
\hline XX Tau & $\mathrm{N}$ & 0.136 & 187 & -12 & 0.268 & 16.652 & 0.808 & -0.015 & 5.47 & 1547 \\
\hline DW UMa & NL & 0.13661 & 150 & 50 & 0.007 & 15.914 & 0.359 & 0.346 & 7.53 & 475 \\
\hline TT Ari & NL & 0.13755 & 149 & -43 & 0.051 & 10.998 & 0.090 & 0.030 & 4.38 & 206 \\
\hline V2289 Cyg & NL & 0.138 & 83 & 15 & 0.061 & 15.858 & -0.150 & 0.609 & 8.00 & 364 \\
\hline V603 Aql & $\mathrm{N}$ & 0.1382 & 33 & 0 & 0.631 & 11.700 & 0.188 & 0.161 & 4.32 & 232 \\
\hline
\end{tabular}


Table 1 - continued

\begin{tabular}{|c|c|c|c|c|c|c|c|c|c|c|}
\hline GCVS-Name & Type & $P$ & $l$ & $b$ & $E(B-V)$ & $J$ & $(J-H)$ & $\left(H-K_{s}\right)$ & $M(J)$ & $d$ \\
\hline DY Pup & $\mathrm{N}$ & 0.139 & 246 & 4 & 0.048 & 14.786 & 0.538 & 0.221 & 6.93 & 365 \\
\hline WX Ari & NL & 0.13935 & 164 & -43 & 0.206 & 14.410 & 0.314 & 0.177 & 5.67 & 514 \\
\hline V1500 Cyg & $\mathrm{N}$ & 0.13961 & 90 & 0 & 0.350 & 16.125 & 0.633 & 0.116 & 5.73 & 1038 \\
\hline TT Tri & NL & 0.13964 & 133 & -32 & 0.060 & 14.608 & 0.219 & 0.118 & 5.31 & 707 \\
\hline V1315 Aql & NL & 0.13969 & 46 & 0 & 0.634 & 14.069 & 0.459 & 0.170 & 5.06 & 489 \\
\hline J0636+3535 & NL & 0.1397 & 179 & 13 & 0.127 & 15.430 & 0.270 & 0.076 & 4.98 & 1167 \\
\hline BY Cam & NL & 0.13975 & 152 & 16 & 0.027 & 13.957 & 0.930 & 0.544 & 10.37 & 52 \\
\hline BO Cet & NL & 0.1398 & 162 & -59 & 0.031 & 14.024 & 0.200 & 0.182 & 5.79 & 438 \\
\hline V909 Sgr & $\mathrm{N}$ & 0.14 & 359 & -10 & 0.087 & 15.117 & 0.568 & -0.035 & 5.02 & 1010 \\
\hline V1223 Sgr & NL & 0.14024 & 5 & -14 & 0.078 & 12.810 & 0.071 & 0.099 & 4.73 & 399 \\
\hline V1432 Aql & NL & 0.14024 & 29 & -16 & 0.076 & 14.592 & 0.472 & 0.422 & 8.16 & 187 \\
\hline V388 Peg & NL & 0.14063 & 67 & -35 & 0.066 & 16.204 & 0.521 & 0.144 & 6.25 & 952 \\
\hline $1813+6122$ & NL & 0.1408 & 91 & 28 & 0.037 & 14.835 & 0.123 & 0.067 & 4.71 & 1044 \\
\hline V849 Her & $\mathrm{CV}$ & 0.1409 & 28 & 35 & 0.073 & 14.951 & 0.192 & 0.101 & 5.06 & 922 \\
\hline MN Hya & NL & 0.14124 & 255 & 19 & 0.054 & 14.851 & 0.510 & 0.221 & 6.81 & 397 \\
\hline AQ Men & NL & 0.14147 & 292 & -31 & 0.181 & 13.926 & 0.230 & 0.206 & 5.69 & 412 \\
\hline V2400 Oph & NL & 0.142 & 0 & 9 & 0.227 & 13.482 & 0.273 & 0.184 & 5.52 & 356 \\
\hline AH Pic & NL & 0.142 & 268 & -30 & 0.043 & 13.899 & 0.178 & 0.089 & 4.98 & 597 \\
\hline V584 Lyr & $\mathrm{DN}$ & 0.1429 & 61 & 9 & 0.167 & 14.998 & 0.460 & -0.047 & 4.42 & 1220 \\
\hline LS Cam & NL & 0.143 & 141 & 22 & 0.162 & 16.124 & 0.059 & 0.527 & 7.62 & 469 \\
\hline $\mathrm{UU} \mathrm{Col}$ & NL & 0.144 & 236 & -34 & 0.021 & 16.902 & 0.643 & 0.391 & 8.43 & 491 \\
\hline V1101 Aql & $\mathrm{DN}$ & 0.1442 & 56 & 56 & 0.021 & 14.898 & 0.434 & 0.041 & 5.30 & 824 \\
\hline LN UMa & NL & 0.1444 & 144 & 43 & 0.081 & 14.735 & 0.079 & 0.222 & 5.58 & 656 \\
\hline V751 Cyg & NL & 0.14446 & 85 & 0 & 0.341 & 13.622 & 0.193 & 0.192 & 5.09 & 443 \\
\hline VZ Scl & NL & 0.14462 & 33 & -74 & 0.020 & 16.093 & 0.619 & 0.301 & 7.70 & 474 \\
\hline RR Pic & $\mathrm{N}$ & 0.14503 & 272 & -26 & 0.046 & 12.458 & 0.060 & 0.144 & 5.02 & 301 \\
\hline CP Lac & $\mathrm{N}$ & 0.14514 & 102 & -1 & 0.294 & 15.025 & 0.291 & -0.080 & 3.42 & 1855 \\
\hline V500 Aql & $\mathrm{N}$ & 0.1452 & 48 & -9 & 0.062 & 13.901 & 0.517 & 0.200 & 6.59 & 283 \\
\hline $\mathrm{J} 2316-0527$ & NL & 0.14545 & 72 & -59 & 0.037 & 15.218 & 0.420 & 0.162 & 6.10 & 656 \\
\hline IM Eri & NL & 0.14562 & 217 & -41 & 0.022 & 11.502 & 0.201 & 0.094 & 5.06 & 192 \\
\hline J1007-2017 & NL & 0.1458 & 259 & 28 & 0.047 & 16.304 & 0.674 & 0.411 & 8.57 & 346 \\
\hline PX And & NL & 0.14635 & 117 & -36 & 0.044 & 14.652 & 0.167 & 0.141 & 5.26 & 742 \\
\hline V533 Her & $\mathrm{N}$ & 0.147 & 69 & 24 & 0.045 & 14.707 & 0.054 & 0.016 & 4.03 & 1339 \\
\hline $0506+7725$ & NL & 0.1477 & 135 & 21 & 0.148 & 16.259 & 0.525 & 0.074 & 5.44 & 1370 \\
\hline $0455+8315$ & NL & 0.14873 & 129 & 24 & 0.065 & 14.430 & 0.147 & 0.200 & 5.56 & 580 \\
\hline $0220+0603$ & NL & 0.1492 & 160 & -50 & 0.053 & 15.382 & 0.249 & 0.327 & 6.78 & 515 \\
\hline BB Dor & NL & 0.14923 & 268 & -33 & 0.047 & 14.322 & 0.233 & 0.036 & 4.60 & 864 \\
\hline VZ Sex & $\mathrm{DN}$ & 0.1493 & 232 & 40 & 0.029 & 14.197 & 0.533 & 0.320 & 7.52 & 214 \\
\hline Tau2 & NL & 0.1495 & 184 & -33 & 0.196 & 14.814 & 0.261 & 0.147 & 5.16 & 787 \\
\hline V425 Cas & NL & 0.1496 & 107 & -6 & 0.178 & 14.134 & 0.156 & 0.123 & 4.75 & 701 \\
\hline AO Psc & NL & 0.14963 & 69 & -53 & 0.066 & 13.459 & 0.154 & 0.217 & 5.68 & 349 \\
\hline LU Cam & NL & 0.15 & 146 & 20 & 0.113 & 15.116 & 0.402 & 0.006 & 4.66 & 1178 \\
\hline J0749-0549 & NL & 0.15 & 225 & 10 & 0.140 & 16.278 & 0.433 & 0.012 & 4.72 & 1932 \\
\hline EF Tuc & NL & 0.15 & 310 & -49 & 0.027 & 13.296 & 0.423 & 0.149 & 5.96 & 290 \\
\hline AB Dra & $\mathrm{DN}$ & 0.152 & 110 & 23 & 0.111 & 13.623 & 0.331 & 0.186 & 5.78 & 354 \\
\hline BP Lyn & NL & 0.15281 & 180 & 42 & 0.013 & 13.854 & 0.108 & 0.219 & 5.65 & 436 \\
\hline V794 Aql & NL & 0.1533 & 40 & -21 & 0.097 & 14.188 & 0.217 & 0.234 & 5.84 & 449 \\
\hline V992 Sco & $\mathrm{N}$ & 0.15358 & 344 & -2 & 0.259 & 15.014 & 0.277 & 0.477 & 7.44 & 295 \\
\hline WY Sge & $\mathrm{N}$ & 0.15364 & 53 & -1 & 0.177 & 15.693 & 0.860 & 0.387 & 8.47 & 259 \\
\hline BZ Cam & NL & 0.15369 & 144 & -24 & 0.053 & 13.363 & 0.208 & 0.121 & 5.08 & 445 \\
\hline LD 317 & NL & 0.154 & 110 & -18 & 0.079 & 12.892 & 0.178 & 0.135 & 5.05 & 359 \\
\hline $2117-5417$ & NL & 0.1545 & 343 & -43 & 0.038 & 13.276 & 0.130 & 0.110 & 4.81 & 485 \\
\hline QQ Vul & NL & 0.15452 & 62 & -5 & 0.200 & 13.580 & 0.337 & 0.187 & 5.56 & 370 \\
\hline PY Per & DN & 0.1548 & 147 & -20 & 0.050 & 14.801 & 0.279 & 0.328 & 6.78 & 394 \\
\hline GS Pav & NL & 0.15527 & 326 & -32 & 0.042 & 14.932 & 0.480 & 0.267 & 6.86 & 405 \\
\hline OY Ara & $\mathrm{N}$ & 0.15547 & 334 & -4 & 0.269 & 15.567 & 0.506 & 0.191 & 5.87 & 781 \\
\hline BH Lyn & NL & 0.15588 & 168 & 35 & 0.040 & 14.812 & 0.208 & 0.176 & 5.48 & 724 \\
\hline SV CMi & DN & 0.156 & 212 & 11 & 0.033 & 14.420 & 0.471 & 0.161 & 6.06 & 463 \\
\hline V1493 Aql & $\mathrm{N}$ & 0.156 & 46 & 2 & 0.044 & 13.555 & 1.126 & 0.376 & 9.33 & 69 \\
\hline
\end{tabular}


Table 1 - continued

\begin{tabular}{|c|c|c|c|c|c|c|c|c|c|c|}
\hline GCVS-Name & Type & $P$ & $l$ & $b$ & $E(B-V)$ & $J$ & $(J-H)$ & $\left(H-K_{s}\right)$ & $M(J)$ & $d$ \\
\hline $0642+5049$ & NL & 0.1569 & 165 & 20 & 0.089 & 14.910 & 0.236 & -0.043 & 3.81 & 1598 \\
\hline V382 Vel & $\mathrm{N}$ & 0.1581 & 284 & 6 & 0.013 & 10.991 & -0.084 & 0.707 & 8.66 & 29 \\
\hline IP Peg & $\mathrm{DN}$ & 0.15821 & 95 & -40 & 0.021 & 12.601 & 0.626 & 0.254 & 7.14 & 123 \\
\hline LX Ser & NL & 0.15843 & 30 & 51 & 0.042 & 13.926 & 0.159 & 0.121 & 4.90 & 629 \\
\hline VY For & NL & 0.1586 & 220 & -54 & 0.015 & 15.589 & 0.589 & 0.130 & 6.14 & 773 \\
\hline CY Lyr & $\mathrm{DN}$ & 0.1591 & 57 & 11 & 0.125 & 13.633 & 0.257 & 0.150 & 5.17 & 467 \\
\hline V380 Oph & NL & 0.16 & 31 & 16 & 0.168 & 14.157 & 0.147 & 0.173 & 4.95 & 648 \\
\hline V4077 Sgr & $\mathrm{N}$ & 0.16 & 7 & -8 & 0.138 & 16.052 & 0.463 & 0.570 & 8.77 & 270 \\
\hline $\mathrm{J} 2337+4308$ & NL & 0.1605 & 109 & -18 & 0.103 & 15.366 & 0.122 & 0.420 & 6.84 & 486 \\
\hline $0229+8016$ & NL & 0.16149 & 127 & 19 & 0.186 & 13.766 & 0.192 & 0.196 & 5.18 & 484 \\
\hline V367 Peg & $\mathrm{DN}$ & 0.1619 & 84 & -36 & 0.057 & 16.423 & 0.481 & 0.492 & 8.39 & 395 \\
\hline CM Del & NL & 0.162 & 59 & -11 & 0.102 & 13.432 & 0.191 & 0.109 & 4.70 & 534 \\
\hline KT Per & $\mathrm{DN}$ & 0.16266 & 130 & -11 & 0.121 & 13.311 & 0.488 & 0.198 & 6.08 & 265 \\
\hline KR Aur & NL & 0.1628 & 184 & 6 & 0.586 & 16.251 & 0.673 & -0.189 & 2.69 & 4059 \\
\hline AR And & $\mathrm{DN}$ & 0.163 & 134 & -23 & 0.047 & 14.589 & 0.593 & 0.266 & 7.01 & 322 \\
\hline CN Ori & $\mathrm{DN}$ & 0.1632 & 211 & -15 & 0.311 & 13.806 & 0.501 & 0.203 & 5.73 & 363 \\
\hline UU Aql & $\mathrm{DN}$ & 0.16353 & 32 & -18 & 0.085 & 14.262 & 0.692 & 0.239 & 6.98 & 277 \\
\hline UU Aqr & NL & 0.16358 & 57 & -45 & 0.132 & 12.971 & 0.342 & 0.146 & 5.28 & 327 \\
\hline X Leo & $\mathrm{DN}$ & 0.1644 & 224 & 45 & 0.021 & 14.287 & 0.404 & 0.289 & 6.73 & 322 \\
\hline V1776 Cyg & NL & 0.16474 & 83 & 5 & 0.912 & 15.272 & 0.406 & 0.001 & 2.65 & 2301 \\
\hline V1193 Ori & NL & 0.165 & 202 & -21 & 0.128 & 13.547 & 0.073 & 0.077 & 4.06 & 748 \\
\hline AM Cas & DN & 0.1652 & 130 & 9 & 0.447 & 12.724 & 0.135 & 0.132 & 3.93 & 479 \\
\hline DO Dra & NL & 0.16537 & 130 & 45 & 0.009 & 13.213 & 0.590 & 0.296 & 7.27 & 154 \\
\hline DO Aql & $\mathrm{N}$ & 0.16776 & 32 & -12 & 0.245 & 16.145 & 0.554 & 0.277 & 6.49 & 773 \\
\hline VW Vul & $\mathrm{DN}$ & 0.1687 & 71 & -13 & 0.132 & 13.524 & 0.250 & 0.106 & 4.67 & 558 \\
\hline $0922+1333$ & NL & 0.169 & 218 & 40 & 0.022 & 13.449 & 0.654 & 0.259 & 7.09 & 186 \\
\hline $0139+0559$ & NL & 0.1692 & 145 & -54 & 0.047 & 14.915 & 0.071 & 0.218 & 5.21 & 857 \\
\hline LZ Mus & $\mathrm{N}$ & 0.1693 & 297 & -3 & 0.157 & 14.455 & 0.707 & 0.241 & 6.79 & 320 \\
\hline J0407-0644 & $\mathrm{DN}$ & 0.17017 & 198 & -39 & 0.086 & 15.204 & 0.496 & -0.003 & 4.58 & 1286 \\
\hline V405 Aur & NL & 0.17262 & 159 & 14 & 0.124 & 13.497 & 0.180 & 0.173 & 4.94 & 489 \\
\hline V849 Oph & $\mathrm{N}$ & 0.17276 & 39 & 13 & 0.101 & 14.683 & 0.617 & 0.182 & 6.19 & 480 \\
\hline UZ Ser & $\mathrm{DN}$ & 0.173 & 15 & 2 & 0.173 & 14.031 & 0.481 & 0.349 & 6.91 & 248 \\
\hline V729 Sgr & $\mathrm{DN}$ & 0.17341 & 12 & -17 & 0.127 & 13.310 & 0.263 & 0.027 & 4.07 & 670 \\
\hline J0704+2625 & NL & 0.174 & 190 & 14 & 0.072 & 16.434 & 0.047 & 0.278 & 5.47 & 1514 \\
\hline V1043 Cen & NL & 0.17459 & 308 & 30 & 0.039 & 12.817 & 0.665 & 0.220 & 6.71 & 164 \\
\hline LS Peg & NL & 0.17477 & 71 & -30 & 0.066 & 11.675 & 0.088 & 0.093 & 4.21 & 303 \\
\hline $\mathrm{J} 2048+0050$ & NL & 0.175 & 48 & -25 & 0.097 & 15.628 & 0.607 & 0.174 & 6.08 & 779 \\
\hline V436 Car & NL & 0.1753 & 266 & -14 & 0.159 & 14.639 & 0.246 & 0.289 & 5.86 & 535 \\
\hline GY Cnc & DN & 0.17544 & 210 & 39 & 0.029 & 13.958 & 0.566 & 0.274 & 6.86 & 259 \\
\hline WW Cet & $\mathrm{DN}$ & 0.1758 & 90 & -71 & 0.030 & 11.075 & 0.144 & 0.108 & 4.53 & 201 \\
\hline CW Mon & DN & 0.1766 & 211 & -3 & 0.112 & 13.874 & 0.534 & 0.319 & 6.91 & 236 \\
\hline U Gem & $\mathrm{DN}$ & 0.17691 & 199 & 23 & 0.019 & 11.651 & 0.584 & 0.239 & 6.65 & 99 \\
\hline V405 Peg & $\mathrm{DN}$ & 0.17764 & 94 & -35 & 0.118 & 12.666 & 0.654 & 0.198 & 6.30 & 179 \\
\hline ES Dra & DN & 0.179 & 98 & 47 & 0.018 & 15.458 & 0.578 & -0.009 & 4.78 & 1358 \\
\hline $\mathrm{J} 2216+2900$ & $\mathrm{DN}$ & 0.1792 & 87 & -23 & 0.049 & 14.445 & 0.546 & 0.417 & 7.77 & 212 \\
\hline BD Pav & DN & 0.1793 & 338 & -22 & 0.058 & 13.467 & 0.459 & 0.110 & 5.25 & 430 \\
\hline GI Mon & $\mathrm{N}$ & 0.1802 & 223 & 5 & 0.107 & 15.334 & 0.317 & 0.205 & 5.46 & 902 \\
\hline V2306 Cyg & NL & 0.1812 & 69 & 2 & 0.748 & 15.069 & 0.365 & -0.043 & 2.34 & 2585 \\
\hline FO Per & $\mathrm{DN}$ & 0.18248 & 151 & 0 & 0.177 & 13.465 & 0.210 & 0.083 & 4.11 & 692 \\
\hline TW Vir & $\mathrm{DN}$ & 0.18267 & 274 & 55 & 0.027 & 13.350 & 0.270 & 0.141 & 5.01 & 460 \\
\hline SS Aur & $\mathrm{DN}$ & 0.1828 & 166 & 13 & 0.062 & 12.701 & 0.433 & 0.267 & 6.29 & 187 \\
\hline MQ Dra & NL & 0.18297 & 86 & 49 & 0.014 & 14.594 & 0.555 & 0.274 & 6.76 & 368 \\
\hline J0649-0737 & NL & 0.183 & 220 & -3 & 0.182 & 15.169 & 0.587 & 0.280 & 6.52 & 498 \\
\hline $0943+1404$ & NL & 0.18416 & 221 & 50 & 0.034 & 15.735 & 0.401 & 0.189 & 5.67 & 1014 \\
\hline Leo7 & DN & 0.1868 & 227 & 40 & 0.037 & 14.927 & 0.564 & 0.273 & 6.67 & 441 \\
\hline V697 Sco & $\mathrm{N}$ & 0.187 & 353 & -5 & 0.155 & 14.037 & 0.629 & 0.227 & 6.25 & 340 \\
\hline $1857+7127$ & DN & 0.18911 & 102 & 25 & 0.068 & 14.739 & 0.419 & 0.226 & 5.86 & 582 \\
\hline CG Dra & DN & 0.1893 & 83 & 19 & 0.052 & 14.783 & 0.522 & 0.231 & 6.18 & 514 \\
\hline AI Tri & NL & 0.19175 & 141 & -30 & 0.061 & 14.210 & 0.286 & 0.300 & 6.03 & 421 \\
\hline
\end{tabular}


Table 1 - continued

\begin{tabular}{|c|c|c|c|c|c|c|c|c|c|c|}
\hline GCVS-Name & Type & $P$ & $l$ & $b$ & $E(B-V)$ & $J$ & $(J-H)$ & $\left(H-K_{s}\right)$ & $M(J)$ & $d$ \\
\hline DQ Her & $\mathrm{N}$ & 0.19362 & 73 & 26 & 0.030 & 13.600 & 0.319 & 0.196 & 5.39 & 433 \\
\hline IX Vel & NL & 0.19393 & 265 & -8 & 0.057 & 9.118 & 0.137 & 0.153 & 4.55 & 80 \\
\hline CT Ser & $\mathrm{N}$ & 0.195 & 24 & 48 & 0.039 & 16.040 & 0.438 & 0.242 & 6.01 & 1000 \\
\hline V433 Ara & NL & 0.19573 & 331 & -9 & 0.086 & 15.798 & 0.497 & 0.455 & 7.62 & 418 \\
\hline MU Cam & NL & 0.19664 & 141 & 24 & 0.095 & 14.341 & 0.225 & 0.263 & 5.47 & 573 \\
\hline UX UMa & NL & 0.19667 & 107 & 64 & 0.011 & 12.758 & 0.349 & 0.145 & 5.10 & 339 \\
\hline $\mathrm{J} 1023+0038$ & NL & 0.19809 & 243 & 46 & 0.050 & 16.296 & 0.603 & -0.169 & 3.34 & 3823 \\
\hline V345 Pav & NL & 0.1981 & 338 & -29 & 0.065 & 12.151 & 0.389 & 0.090 & 4.66 & 307 \\
\hline V895 Cen & NL & 0.19855 & 323 & 21 & 0.062 & 13.975 & 0.560 & 0.268 & 6.42 & 316 \\
\hline HX Peg & $\mathrm{DN}$ & 0.2008 & 97 & -47 & 0.085 & 13.224 & 0.228 & 0.070 & 4.02 & 671 \\
\hline $\mathrm{AT} \mathrm{Cnc}$ & $\mathrm{DN}$ & 0.2011 & 199 & 31 & 0.038 & 12.482 & 0.154 & 0.092 & 4.09 & 470 \\
\hline FO Aqr & NL & 0.20206 & 53 & -49 & 0.052 & 12.872 & 0.127 & 0.237 & 5.04 & 361 \\
\hline $\mathrm{T}$ Aur & $\mathrm{N}$ & 0.20438 & 177 & -2 & 0.232 & 14.016 & 0.277 & 0.162 & 4.46 & 741 \\
\hline J0911+0841 & DN & 0.2054 & 222 & 35 & 0.062 & 15.332 & 0.628 & 0.189 & 5.93 & 740 \\
\hline V825 Her & NL & 0.206 & 66 & 34 & 0.029 & 13.879 & 0.279 & 0.070 & 4.21 & 848 \\
\hline V446 Her & $\mathrm{N}$ & 0.207 & 45 & 5 & 0.453 & 15.387 & 0.586 & 0.108 & 4.35 & 1342 \\
\hline V3885 Sgr & NL & 0.20714 & 357 & -28 & 0.024 & 9.955 & 0.220 & 0.119 & 4.42 & 127 \\
\hline V617 Sgr & NL & 0.20717 & 357 & -7 & 0.121 & 13.786 & 0.267 & 0.247 & 5.27 & 480 \\
\hline TW Tri & $\mathrm{DN}$ & 0.20758 & 134 & -30 & 0.038 & 14.565 & 0.575 & 0.268 & 6.40 & 423 \\
\hline V4745 Sgr & $\mathrm{N}$ & 0.20782 & 1 & -12 & 0.108 & 15.446 & -0.003 & 0.314 & 5.08 & 1132 \\
\hline RX And & $\mathrm{DN}$ & 0.20989 & 126 & -21 & 0.028 & 12.454 & 0.707 & 0.187 & 6.14 & 181 \\
\hline EX Dra & DN & 0.20994 & 98 & 29 & 0.027 & 12.882 & 0.617 & 0.205 & 6.04 & 231 \\
\hline J0808+3131 & $\mathrm{DN}$ & 0.21 & 190 & 29 & 0.042 & 16.178 & 0.515 & 0.118 & 5.10 & 1617 \\
\hline HZ Pup & $\mathrm{N}$ & 0.213 & 246 & 1 & 0.371 & 15.910 & 0.275 & -0.022 & 2.69 & 3788 \\
\hline AP Cru & $\mathrm{N}$ & 0.213 & 301 & -2 & 0.250 & 15.457 & 0.696 & 0.276 & 6.25 & 628 \\
\hline PW Vul & $\mathrm{N}$ & 0.2137 & 61 & 5 & 0.226 & 16.140 & 0.643 & 0.312 & 6.42 & 801 \\
\hline HR Del & $\mathrm{N}$ & 0.21417 & 63 & -14 & 0.106 & 12.323 & 0.048 & 0.056 & 3.24 & 627 \\
\hline PQ Gem & NL & 0.21636 & 206 & 20 & 0.025 & 13.494 & 0.293 & 0.205 & 5.13 & 465 \\
\hline HL CMa & $\mathrm{DN}$ & 0.21679 & 227 & -8 & 0.168 & 11.637 & 0.188 & 0.216 & 4.62 & 237 \\
\hline AY Psc & DN & 0.21732 & 142 & -54 & 0.050 & 14.517 & 0.500 & 0.015 & 4.20 & 1134 \\
\hline CZ Ori & DN & 0.2189 & 195 & 0 & 0.107 & 12.587 & 0.227 & 0.061 & 3.68 & 578 \\
\hline $\mathrm{J} 2243+3055$ & $\mathrm{DN}$ & 0.21894 & 93 & -24 & 0.064 & 12.874 & 0.091 & 0.057 & 3.40 & 766 \\
\hline $\mathrm{J} 0900+4301$ & $\mathrm{DN}$ & 0.221 & 178 & 41 & 0.020 & 15.746 & 0.681 & 0.092 & 5.26 & 1241 \\
\hline V709 Cas & NL & 0.2225 & 120 & -3 & 0.112 & 13.136 & 0.360 & 0.266 & 5.50 & 322 \\
\hline EZ Del & DN & 0.2234 & 58 & -12 & 0.182 & 15.083 & 0.298 & 0.076 & 3.77 & 1701 \\
\hline V4742 Sgr & $\mathrm{N}$ & 0.225 & 5 & -1 & 0.519 & 14.458 & 0.442 & 0.283 & 4.91 & 656 \\
\hline V705 Cas & $\mathrm{N}$ & 0.228 & 114 & -4 & 0.221 & 14.884 & 0.349 & 0.259 & 5.12 & 820 \\
\hline TV Col & NL & 0.2286 & 237 & -30 & 0.024 & 13.197 & 0.369 & 0.136 & 4.69 & 499 \\
\hline WX Pyx & NL & 0.2307 & 245 & 10 & 0.077 & 15.313 & 0.361 & 0.119 & 4.40 & 1477 \\
\hline RW Tri & NL & 0.23188 & 147 & -30 & 0.077 & 11.938 & 0.360 & 0.118 & 4.38 & 316 \\
\hline V347 Pup & NL & 0.23194 & 256 & -27 & 0.036 & 13.129 & 0.648 & 0.192 & 5.75 & 294 \\
\hline VY Scl & NL & 0.2323 & 20 & -72 & 0.021 & 12.839 & 0.049 & 0.091 & 3.48 & 737 \\
\hline J0732-1331 & NL & 0.2335 & 230 & 3 & 0.079 & 12.906 & 0.369 & 0.082 & 4.12 & 555 \\
\hline DO Leo & NL & 0.23452 & 228 & 57 & 0.037 & 15.926 & 0.731 & -0.021 & 4.37 & 2016 \\
\hline TX Col & NL & 0.2383 & 247 & -30 & 0.034 & 13.633 & 0.264 & 0.201 & 4.77 & 585 \\
\hline AH Eri & DN & 0.2391 & 208 & -38 & 0.091 & 15.923 & 0.577 & 0.401 & 6.92 & 609 \\
\hline M 5-V101 & DN & 0.242 & 4 & 47 & 0.045 & 15.656 & 0.358 & 0.426 & 6.61 & 633 \\
\hline RW Sex & NL & 0.24507 & 252 & 39 & 0.033 & 10.321 & 0.176 & 0.075 & 3.54 & 224 \\
\hline V1039 Cen & $\mathrm{N}$ & 0.247 & 310 & -2 & 0.207 & 15.886 & 0.080 & 0.732 & 7.74 & 392 \\
\hline LL Lyr & $\mathrm{DN}$ & 0.24907 & 67 & 19 & 0.068 & 15.424 & 0.528 & 0.248 & 5.62 & 890 \\
\hline RU LMi & DN & 0.251 & 192 & 53 & 0.012 & 16.684 & 0.285 & 0.593 & 7.63 & 643 \\
\hline V1425 Aql & $\mathrm{N}$ & 0.2558 & 33 & -4 & 0.293 & 14.538 & 0.476 & 0.202 & 4.58 & 871 \\
\hline AH Her & $\mathrm{DN}$ & 0.25812 & 45 & 38 & 0.032 & 11.806 & 0.332 & 0.099 & 3.99 & 360 \\
\hline FY Per & NL & 0.2585 & 155 & 3 & 0.190 & 11.747 & 0.353 & 0.099 & 3.70 & 377 \\
\hline TZ Per & $\mathrm{DN}$ & 0.26291 & 134 & -3 & 0.231 & 13.087 & 0.580 & 0.112 & 4.26 & 531 \\
\hline TW Pic & NL & 0.265 & 266 & -33 & 0.056 & 14.776 & 0.433 & 0.227 & 5.09 & 848 \\
\hline BV Pup & DN & 0.265 & 240 & 1 & 0.089 & 13.336 & 0.447 & 0.099 & 4.10 & 677 \\
\hline U Leo & $\mathrm{N}$ & 0.2674 & 226 & 53 & 0.042 & 16.212 & 0.405 & 0.271 & 5.34 & 1469 \\
\hline EI UMa & DN & 0.2681 & 171 & 37 & 0.031 & 13.866 & 0.277 & 0.055 & 3.43 & 1206 \\
\hline
\end{tabular}


Table 1 - continued

\begin{tabular}{|c|c|c|c|c|c|c|c|c|c|c|}
\hline GCVS-Name & Type & $P$ & $l$ & $b$ & $E(B-V)$ & $J$ & $(J-H)$ & $\left(H-K_{s}\right)$ & $M(J)$ & $d$ \\
\hline TT Crt & DN & 0.26842 & 275 & 47 & 0.028 & 13.874 & 0.480 & 0.208 & 5.10 & 563 \\
\hline CM Phe & NL & 0.2689 & 314 & -65 & 0.011 & 13.047 & 0.581 & 0.229 & 5.54 & 315 \\
\hline LY Uma & DN & 0.27128 & 157 & 56 & 0.010 & 13.102 & 0.614 & 0.139 & 4.95 & 426 \\
\hline SS Cyg & DN & 0.27513 & 91 & -7 & 0.055 & 8.516 & 0.160 & 0.057 & 3.03 & 122 \\
\hline $2347-3144$ & NL & 0.277 & 11 & -76 & 0.013 & 16.135 & 0.602 & -0.013 & 3.74 & 2999 \\
\hline BY Cir & $\mathrm{N}$ & 0.2816 & 315 & -3 & 0.613 & 15.663 & 0.300 & 0.391 & 4.58 & 1281 \\
\hline V426 Oph & DN & 0.2853 & 33 & 12 & 0.051 & 10.997 & 0.502 & 0.166 & 4.64 & 183 \\
\hline $\mathrm{J} 0813+4528$ & $\mathrm{DN}$ & 0.289 & 174 & 33 & 0.049 & 15.937 & 0.684 & 0.106 & 4.64 & 1780 \\
\hline Z Cam & DN & 0.28984 & 141 & 32 & 0.020 & 11.571 & 0.526 & 0.189 & 4.90 & 214 \\
\hline EM Cyg & DN & 0.29091 & 65 & 4 & 0.106 & 11.735 & 0.402 & 0.183 & 4.34 & 289 \\
\hline V838 Her & $\mathrm{N}$ & 0.29764 & 43 & 7 & 0.406 & 16.129 & 0.666 & 0.104 & 3.73 & 2559 \\
\hline $\mathrm{J} 2133+5107$ & NL & 0.2997 & 94 & -1 & 0.253 & 13.884 & 0.337 & 0.089 & 3.08 & 1306 \\
\hline V2274 Cyg & $\mathrm{N}$ & 0.3 & 73 & 2 & 0.526 & 16.120 & 0.441 & 0.494 & 5.74 & 961 \\
\hline $\mathrm{AC} \mathrm{Cnc}$ & NL & 0.30048 & 214 & 31 & 0.036 & 13.078 & 0.383 & 0.102 & 3.76 & 720 \\
\hline RY Ser & $\mathrm{DN}$ & 0.3009 & 11 & 13 & 0.314 & 13.699 & 0.631 & 0.181 & 4.38 & 642 \\
\hline V2069 Cyg & NL & 0.31168 & 87 & -6 & 0.449 & 14.347 & 0.431 & 0.182 & 3.48 & 1238 \\
\hline V2275 Cyg & $\mathrm{N}$ & 0.3145 & 89 & 1 & 0.071 & 16.246 & 0.220 & 1.037 & 10.05 & 169 \\
\hline HQ Mon & NL & 0.316 & 214 & 5 & 0.117 & 12.645 & 0.165 & 0.110 & 2.96 & 826 \\
\hline V363 Aur & NL & 0.32124 & 172 & 2 & 0.344 & 13.295 & 0.332 & 0.160 & 3.22 & 901 \\
\hline V392 Нya & DN & 0.32495 & 275 & 28 & 0.051 & 14.810 & 0.661 & 0.097 & 4.22 & 1283 \\
\hline V1309 Ori & NL & 0.33261 & 200 & -21 & 0.129 & 14.223 & 0.561 & 0.150 & 4.12 & 995 \\
\hline BT Mon & $\mathrm{N}$ & 0.33381 & 214 & -3 & 0.438 & 14.399 & 0.442 & 0.236 & 3.77 & 1119 \\
\hline MU Cen & DN & 0.342 & 296 & 17 & 0.095 & 13.029 & 0.507 & 0.237 & 4.63 & 460 \\
\hline $\mathrm{CH}$ UMa & DN & 0.34318 & 143 & 43 & 0.061 & 12.711 & 0.505 & 0.166 & 4.17 & 499 \\
\hline V368 Aql & $\mathrm{N}$ & 0.3452 & 44 & -4 & 0.485 & 14.531 & 0.383 & 0.114 & 2.52 & 2068 \\
\hline GY Hya & DN & 0.34724 & 329 & 31 & 0.096 & 13.583 & 0.542 & 0.113 & 3.76 & 885 \\
\hline J2256-2743 & $\mathrm{DN}$ & 0.3501 & 25 & -65 & 0.035 & 15.419 & 0.514 & 0.144 & 4.04 & 1861 \\
\hline HD 45166 & $\mathrm{CV}$ & 0.357 & 203 & -2 & 0.044 & 9.812 & 0.061 & 0.181 & 3.06 & 220 \\
\hline QZ Aur & $\mathrm{N}$ & 0.3575 & 174 & -1 & 0.100 & 15.829 & 0.543 & 0.315 & 5.18 & 1294 \\
\hline RZ Gru & NL & 0.36 & 353 & -60 & 0.014 & 11.938 & 0.263 & 0.120 & 3.18 & 561 \\
\hline RU Peg & $\mathrm{DN}$ & 0.3746 & 74 & -35 & 0.054 & 11.069 & 0.441 & 0.164 & 3.78 & 281 \\
\hline AT Ara & $\mathrm{DN}$ & 0.3755 & 344 & -6 & 0.117 & 13.500 & 0.561 & 0.422 & 5.86 & 322 \\
\hline SY Cnc & $\mathrm{DN}$ & 0.38 & 210 & 36 & 0.027 & 11.273 & 0.222 & 0.129 & 2.98 & 451 \\
\hline J0527-6954 & $\mathrm{CV}$ & 0.3926 & 281 & -33 & 0.075 & 15.588 & 0.733 & -0.079 & 2.59 & 3865 \\
\hline NY Lup & NL & 0.411 & 332 & 7 & 0.247 & 13.225 & 0.477 & 0.220 & 3.64 & 747 \\
\hline AE Aqr & NL & 0.41166 & 45 & -24 & 0.043 & 9.459 & 0.542 & 0.144 & 3.69 & 140 \\
\hline V1062 Tau & NL & 0.41284 & 178 & -10 & 0.415 & 14.324 & 0.639 & 0.449 & 5.37 & 522 \\
\hline WX Cen & NL & 0.41696 & 305 & -1 & 0.161 & 11.863 & 0.342 & 0.361 & 4.48 & 281 \\
\hline $0928+5004$ & NL & 0.41838 & 168 & 46 & 0.013 & 15.653 & 0.252 & 0.112 & 2.73 & 3826 \\
\hline Q Cyg & $\mathrm{N}$ & 0.4202 & 90 & -7 & 0.566 & 13.541 & 0.292 & 0.144 & 1.84 & 1736 \\
\hline KO Vel & NL & 0.422 & 278 & 7 & 0.178 & 16.649 & 0.703 & 0.282 & 4.77 & 2213 \\
\hline DX And & $\mathrm{DN}$ & 0.4405 & 108 & -16 & 0.113 & 13.106 & 0.594 & 0.122 & 3.34 & 858 \\
\hline CAL 87 & $\mathrm{CV}$ & 0.44268 & 282 & -31 & 0.075 & 16.389 & 0.571 & 0.053 & 2.84 & 4970 \\
\hline QU Car & NL & 0.454 & 294 & 1 & 0.137 & 10.972 & 0.124 & 0.141 & 2.13 & 554 \\
\hline UY Pup & DN & 0.47927 & 231 & 6 & 0.187 & 14.145 & 0.514 & 0.136 & 2.87 & 1670 \\
\hline $\mathrm{J} 1951+3716$ & NL & 0.492 & 72 & 5 & 0.209 & 13.250 & 0.550 & 0.147 & 2.93 & 1065 \\
\hline V Sge & $\mathrm{CV}$ & 0.5142 & 62 & -9 & 0.070 & 10.315 & 0.202 & 0.302 & 3.36 & 240 \\
\hline BV Cen & $\mathrm{DN}$ & 0.61011 & 309 & 7 & 0.220 & 11.290 & 0.404 & 0.205 & 2.42 & 544 \\
\hline CI Aql & NR & 0.61836 & 32 & -1 & 0.139 & 13.668 & 0.339 & 0.644 & 5.63 & 382 \\
\hline J1730-0559 & NL & 0.6425 & 18 & -15 & 0.108 & 14.384 & 0.526 & 0.211 & 2.89 & 1904 \\
\hline V723 Cas & $\mathrm{N}$ & 0.69327 & 125 & -9 & 0.091 & 11.882 & 0.196 & 0.755 & 5.90 & 152 \\
\hline
\end{tabular}

star. At first, a total interstellar absorption within the Galaxy in the photometric $V$ band was computed from an available modeled value of $E_{\infty}(B-V)$ for a given galactic latitude $(b)$ as

$$
A_{\infty}(b)=3.1 E_{\infty}(B-V)
$$


which could be reduced to give interstellar absorption in the $V$ band up to the distance $d$ of a CV as following (Bahcall \& Soneira, 1980)

$$
A_{d}(b)=A_{\infty}(b)\left[1-\exp \left(\frac{-|d \sin (b)|}{H}\right)\right]
$$

where $H$ is the scaleheight for the interstellar dust which is adopted to be 100 pc as usual (see e.g. Mendez \& van Altena, 1998). However, the distance of the CV is needed, but it is unknown at this point. Nevertheless, a first order approximation of the distance $d$, which is according to the PLCs relation of Ak et al. (2007) relying on the observed $(J-H)$ and $\left(H-K_{s}\right)$ colours in Table 1 instead of true intrinsic colours, could be used in Eq. 2 for a first order estimate of the interstellar extinction in the $V$ band. This extinction, then, is converted to an $E_{d}(B-V)$ value as following

$$
E_{d}(B-V)=A_{d}(b) / 3.1
$$

which gives the first order approximation of the colour excess $E(B-V)$ for the associated $\mathrm{CV}$. The predicted colour excess $E(B-V)$ values by this method are given in the column 6 of Table 1 . Once, an approximated $E(B-V)$ value is available for a $\mathrm{CV}$, then the interstellar extinctions at $J, H$ and $K_{s}$ bands for it could be computed as $A_{J}=0.887 \times E(B-V), A_{H}=0.565 \times E(B-V)$ and $A_{K_{s}}=0.382 \times E(B-V)$ according to the equations of Fiorucci \& Munari (2003) (see also, Bilir, Güver \& Aslan, 2006). After computing the interstellar extinctions $A_{J}, A_{H}$ and $A_{K_{s}}$, the de-reddened colours $(J-H)_{0}$ and $\left(H-K_{s}\right)_{0}$ could be obtained from $J_{0}=J-A_{J}, H_{0}=H-A_{H}$ and $\left(K_{s}\right)_{0}=K_{s}-A_{K_{s}}$. Those intrinsic colours and the orbital periods (column 3 ) were then used for computing the absolute magnitudes in column 10 of Table 1 by the PLCs relation of CVs.

\subsection{Accuracy and limitations of the PLCs relation}

The PLCs relation was established as a useful tool for predicting CV distances easily from the orbital periods and $2 M A S S$ observations. After discovered by

Ak et al. (2007) as an idea, it was calibrated and tested by the most reliable trigonometric parallaxes of $27 \mathrm{CVs}$ with $\sigma_{\pi} / \pi<0.40$. Therefore, it is especially preferred for this study over the other CV distance indicators because of its consistency, tested reliability and wide ranges of its aplicability. The open form of the PLCs relation is

$$
M_{J}=-0.894-5.721 \log P_{\text {orb }}+2.598(J-H)_{0}+7.380(H-K)_{0},
$$




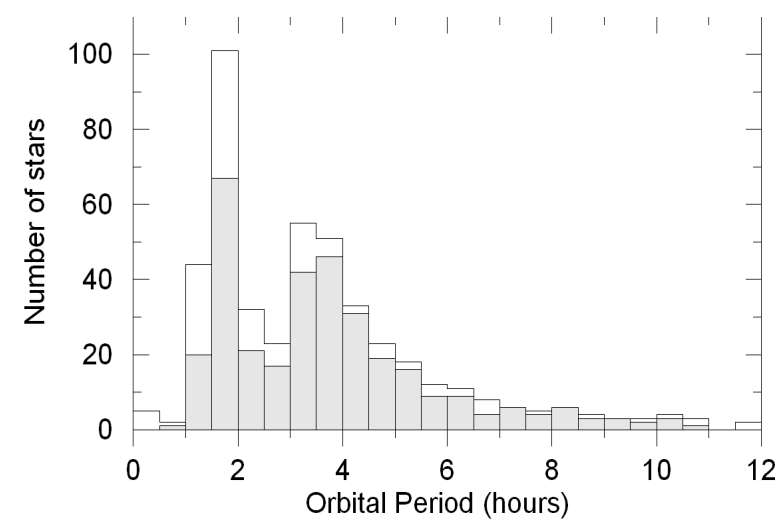

Fig. 1. The orbital period distribution of CVs in the sample.

which uses intrinsic $(J-H)_{0}$ and $\left(H-K_{s}\right)_{0}$ colours and orbital periods to predict absolute magnitude of a $\mathrm{CV}$. The relation was claimed to provide absolute magnitudes within the accuracy of about $\pm 0.22 \mathrm{mag}$ and valid for the ranges; $0.032^{d}<P_{\text {orb }} \leq 0.454^{d},-0.08<(J-H)_{0} \leq 1.54,-0.03<$ $\left(H-K_{s}\right)_{0} \leq 0.56$ and $2.0<M_{J}<11.7$, covering the present data very well. Only 15 per cent of $\mathrm{CVs}$ in the preliminary list is removed because of these limits when forming the list of CVs in Table 1.

Once absolute magnitudes in the $J$ band are available, the distances were then calculated by the Pogson's relation as $d(p c)=10^{\left(J-M_{J}+5-A_{J}\right) / 5}$, where $J$ is observed apparent magnitude and $A_{J}$ is the interstellar absorption in the $J$ band. The distances of CVs in our sample, which are computed by the intrinsic colours, are listed in the last column of Table 1.

The intrinsic colours used in the PLCs relation have been computed as a first order estimation as described. Refinements have not been attempted since a possible correction term and its effect on the predicted distance would be too small and expected to be lost within about 10 per cent uncertainty which is equavalent to $\pm 0.22 \mathrm{mag}$ in the magnitude scale.

\subsection{Completeness of the sample}

Disagreements between the observational and theoretical studies of CV populations could be often attributed to the incompleteness of surveys, which are mostly due to unavoidable selection effects of observational data (Della Valle \& Livio, 1996; Aungwerojwit et al., 2005; Gänsicke, 2005; Pretorius, Knigge \& Kolb, 2007). Indeed, one of the goals of CV surveys is to reduce the selection effects by observing the fainter CVs and thereby provide a more accurate picture of the actual CV population since the theory predicts the most of CVs should be intrinsically faint (Howell, Rappaport \& Politano, 1997; Kolb, 2001). It is obvious that the apparent magnitude introduces a strong bias in a CV sample 
as the detection of faint stars is difficult. Moreover, variability and amplitude of the variability are important factors which may affect the discovering probability of a CV since rare and low-amplitude erupters are harder to discover (Patterson, 1998; Pretorius, Knigge \& Kolb, 2007). Thus, low-mass transfer rate systems are likely under-represented in the known samples of CVs because the intrinsic brightness and eruption frequency decrease with the mass transfer rate.

Nevertheless, the strongest bias in the present sample may appear to be originating from the chance of finding CVs in the $2 M A S S$ observations. Because this bias mostly depends on the apparent magnitudes, it should not be counted as a new kind of bias on the studies which aim to find the space densities in the solar neighbourhood. This is because the systems fainter than the limiting magnitude of the $2 M A S S$ database are expected to be very distant objects. The limiting magnitudes of the 2MASS survey are 15.8, 15.1 and 14.3 in $J$, $H$ and $K_{s}$ bands, respectively (Skrutskie et al., 2006). Therefore, we had to remove 114 systems from our primary sample, which was including more than 640 systems, as they do not exist in the $2 M A S S$ database mostly because they are dimmer than the limiting magnitudes. Additionally, we had to remove 46 systems more from the preliminary sample due to the application limits of the PLCs relation concerned with the de-reddened colour indices $(J-H)_{0}$ and $\left(H-K_{s}\right)_{0}$, as well (see Section 2.2).

Another important bias in our sample is introduced by the orbital periods. Although this bias is magnitude dependent too, it could be counted as one of the biases that stands out independently because it is easier to measure shorter periods. The selection effects due to the orbital periods are more pronounced for magnetic systems since these objects are preferred in the follow-up observations to measure the orbital period (Pretorius, Knigge \& Kolb, 2007). It should be noted that the upper and lower application limits of the PLCs relation concerned with the orbital period (see Section 2.2) do not introduce an additional bias in the CV sample of this study, because almost all CVs with known orbital periods are included in the period limits of the PLCs relation.

Only 24 stars were removed because of the period limits of the PLCs relation. The CVs discovered in globular clusters were removed from the sample, as well. After these removing processes, the number of systems in the final sample drops to 459. Table 1 contains all of the CVs in the final sample. The orbital period distribution of CVs in the final sample is shown in Figure 1. Shaded and white areas in Figure 1 show the samples with the limiting apparent magnitudes of $J_{0}=15.8$ and 16.5, respectively. Among the all systems (459), 8 are CVs with unknown type, 202 dwarf novae, 57 novae, 3 recurrent novae and 189 nova-like stars.

Although the CV sample in this study is not free from the selection effects, 


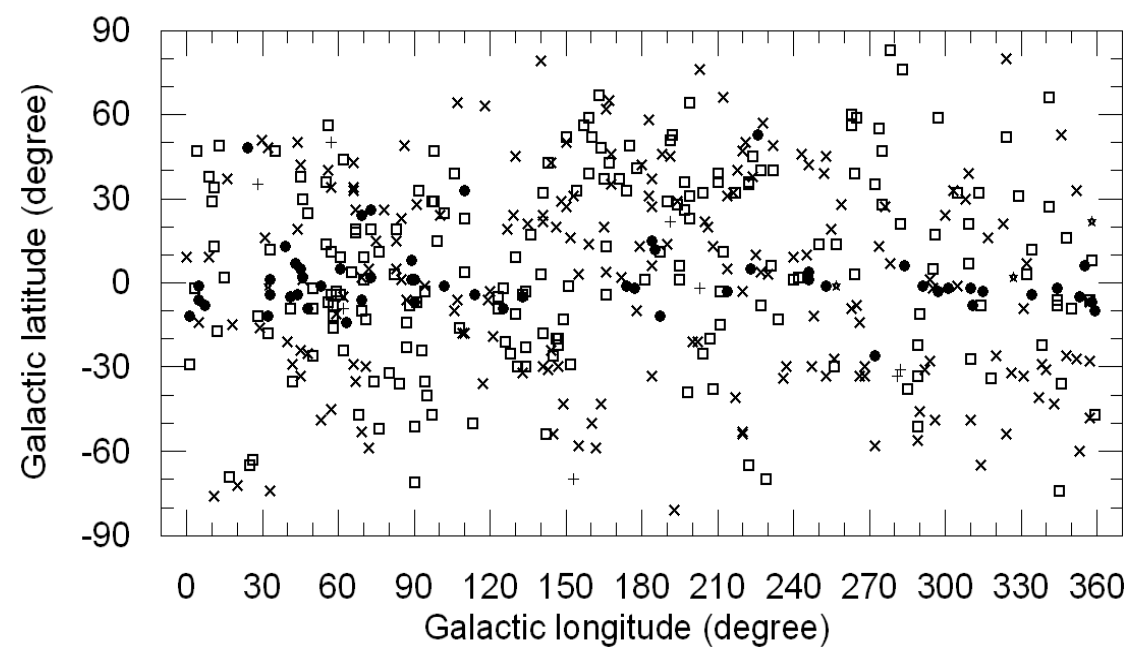

Fig. 2. The galactic distribution of CVs in the sample. The symbol ( $\square$ ) denotes dwarf novae, $(\times)$ nova-like stars, $(\bullet)$ novae, $(\star)$ recurrent novae and $(+)$ CVs with unknown type.

it is the largest sample which has ever been appeared in the literature. Our sample includes $\sim 72$ per cent of CVs with known orbital periods. Thus, space densities of CVs in the solar neighbourhood derived from this sample should be inspiring as well as being useful for constraining the existing population models.

\subsection{Galactic model parameters}

Exponential functions have usually been used to derive the galactic model parameters of the galactic disc. However, a recent study has shown that the observed vertical distribution in the Galaxy is smoother in the solar neighbourhood and it is well-approximated by a secans hiperbolicus function (Bilir et al., 2006).

Therefore, in order to derive scaleheights of CV populations in the solar neighbourhood, we have preferred to test following two functions

$$
n(z)=n_{0} \exp \left(-\frac{|z|}{H}\right),
$$

and

$$
n(z)=n_{0} \operatorname{sech}^{2}\left(-\frac{|z|}{H_{z}}\right),
$$

for describing the number density variation of CVs by the distance from the 


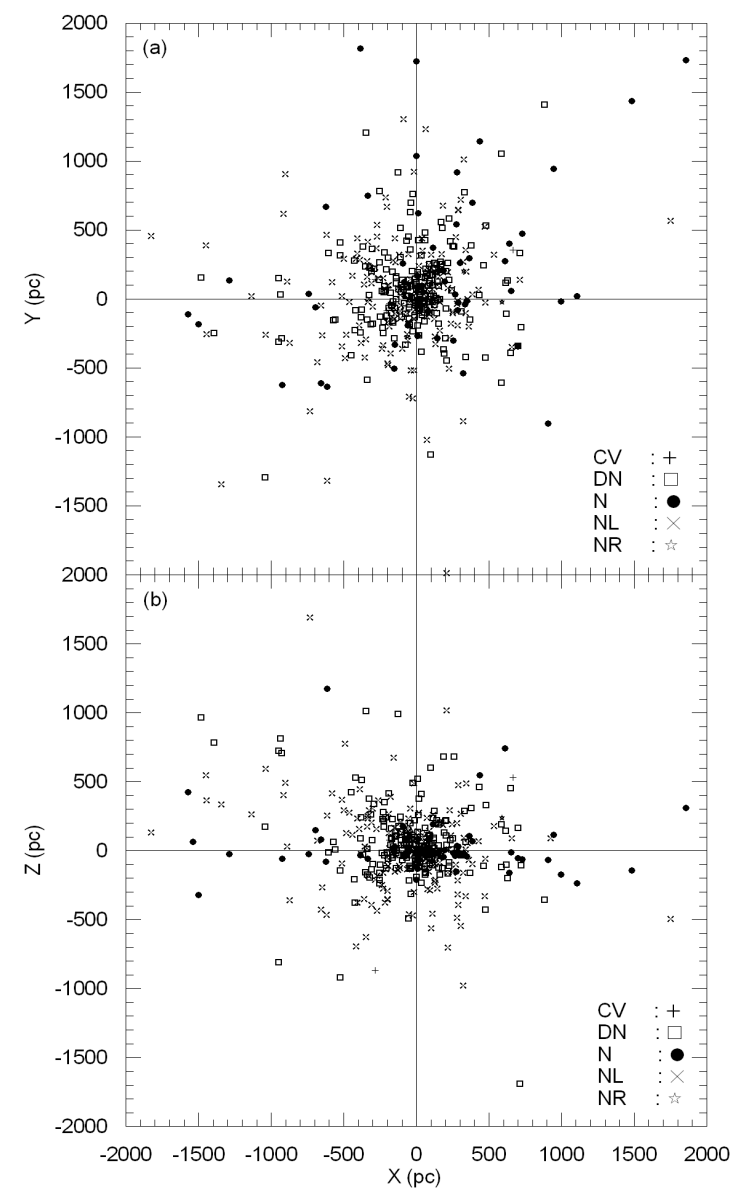

Fig. 3. The spatial distribution of systems in the sample with respect to the Sun. $X$, $Y$ and $Z$ are heliocentric galactic coordinates directed towards the Galactic Centre, galactic rotation and the north Galactic Pole, respectively. CV denotes CVs with unknown types, DN dwarf novae, NL nova-like stars, $\mathrm{N}$ novae and NR recurrent novae.

galactic disc. When choosing the best function to describe actual CV distribution in the solar neigbourhood, the galactic model parameters $\left(n_{0}\right.$ and a scaleheight) were determined for both of the functions first and then their fits, that is, abilities of describing actual distribution are compared by a minimum $\chi^{2}$ test. Consequently, $n_{0}$ is the number density of CVs in the galactic plane, $z$ is the distance from the galactic plane and is given by $z=z_{0}+d \sin (b)$, where $b$ is the galactic latitude of the CV and $z_{0}$ is the distance of Sun from the galactic plane (24 pc, Jurić et al., 2005). $H$ and $H_{z}$ are the exponential and sech $^{2}$ vertical scaleheights, respectively. The relation between the exponential scaleheight $H$ and the $\operatorname{sech}^{2}$ scaleheight $H_{z}$ is $H=1.08504 H_{z}$ (Bilir et al., 2006). Because recent surveys show that the radial scalelength of the thin disc stars is longer than $2.25 \mathrm{kpc}$ (Jurić et al., 2005), we have not attempted to estimate the radial scalelength of CVs since 95 per cent of systems in our sample are closer than $\sim 1 \mathrm{kpc}$ in the $X-Y$-plane (see Figure 3). All error estimates in the analysis were obtained by changing galactic model parameters until an 
Table 2

The numbers, median distances and median heliocentric galactic distances of CVs in the sample. Distances $(d)$ and positions $(X, Y, Z)$ are in pc. CV denotes CVs with unknown types, ALL all systems in the sample, DN dwarf novae, NL nova-like stars, $\mathrm{N}$ novae and NR recurrent novae.

\begin{tabular}{lccccc}
\hline Type & Number & $d$ & $X$ & $Y$ & $Z$ \\
\hline CV & 8 & 581 & 80 & 19 & -23 \\
DN & 202 & 294 & -3 & 50 & 12 \\
NL & 189 & 432 & -43 & 0 & 20 \\
N & 57 & 628 & 114 & 50 & -11 \\
NR & 3 & 278 & 62 & -40 & -5 \\
ALL & 459 & 377 & 0 & 30 & 9 \\
\hline
\end{tabular}

increase or decrease by $2 \sigma$ in $\chi^{2}$ was achieved (Press et al., 1997).

\section{Analysis}

\subsection{Spatial distribution}

The galactic coordinates $(l, b)$ of $\mathrm{CVs}$ in the sample are plotted in Figure 2. The figure indicates that CVs in general all distributed about the galactic plane symmetrically, except for novae which appear concentrated only in the galactic plane.

In order to inspect the spatial distribution of the present CV sample of all systems, the Sun centered rectangular galactic coordinates ( $X$ towards Galactic Center, $Y$ galactic rotation, $Z$ north Galactic Pole) were calculated. The projected positions on the galactic plane ( $X, Y$ plane) and on the plane perpendicular to it ( $X, Z$ plane) are displayed in Figure 3. Having 231 systems with $X \leq 0$ and 228 system with $X>0,207$ systems with $Y \leq 0$ and 252 system with $Y>0,219$ systems with $Z \leq 0$ and 240 system with $Z>0$, we could conclude that there is not a considerable bias introduced by the projected positions of these systems. The numbers, median distances and median heliocentric galactic distances of CVs are listed in Table 2. 


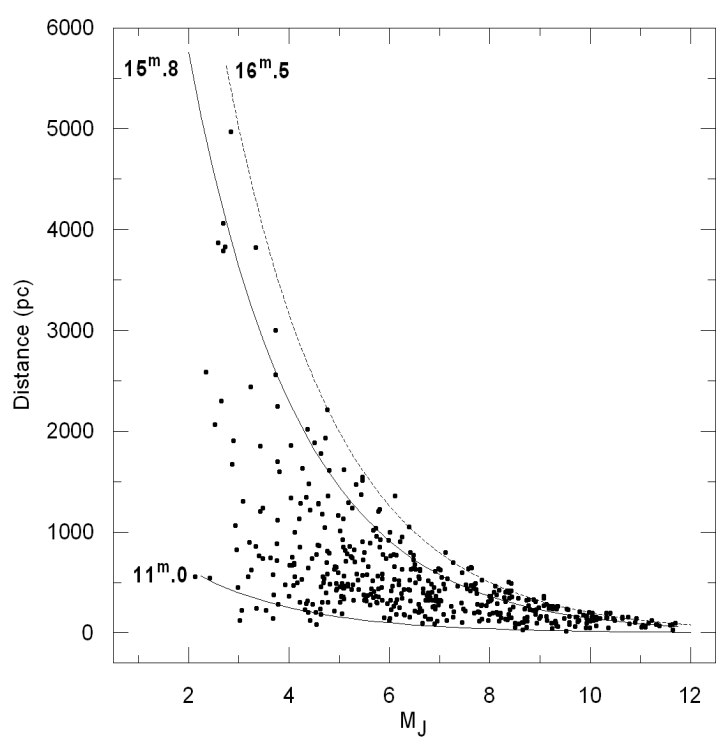

Fig. 4. Distances to $459 \mathrm{CVs}$ plotted versus $M_{J}$. The distance limits for bright $\left(J_{0}=11.0\right)$ and faint $\left(J_{0}=15.8\right.$ and 16.5$)$ apparent magnitudes are shown by lines.

\subsection{Galactic model parameters and space density of $C V s$}

The completeness limits of the sample according to the apparent magnitudes should be estimated before deriving the space density of CVs. Therefore, Figure 4 was drawn to see the $\mathrm{CVs}$ distances versus the absolute magnitudes $M_{J}$ and then for estimating the completeness limits for bright and faint limiting apparent magnitudes in the $J$ band.

The bright apparent magnitude limit of our sample is consistent with $J_{0}=11.0$. In order to take into account the sample's incompleteness towards fainter apparent magnitudes, we assumed two different magnitudes, i.e. $J_{0}=15.8$ and 16.5. The limiting magnitude of 15.8 corresponds to the completeness limit of $J$ band apparent magnitudes for 2MASS observations (Skrutskie et al., 2006), while the limiting magnitude of 16.5 represents almost the whole sample. With the limiting apparent magnitude of $J_{0}=15.8$, the number of systems drops to 354. That is, the size of the sample reduces to 77 per cent of the original size. Consequently, the space densities and galactic model parameters of CVs should be examined for both of the limiting apparent magnitudes in order to see how complete the present sample is.

The first step of doing this is to prepare $z$-histograms, where $z$ is the distance of CVs from the galactic plane computed by equation $z=z_{0}+d \sin |b|$ and binned for per 100 pc intervals. The histograms and the best fits of the exponential and $\operatorname{sech}^{2}$ functions are shown in Figures 5 and 6 . The galactic model parameters obtained from the minimum $\chi^{2}$ analysis are given in Table 3 for two different faint apparent magnitude limits, i.e. 15.8 and 16.5. 
Table 3

The galactic model parameters and their errors for CVs. The parameters are given for two different apparent magnitude limits. ALL denotes all CVs in the sample, DN dwarf novae, NL nova-like stars including polars, AM polars (magnetic systems) and NM non-magnetic CVs (all CVs except polars). $n_{0}$ is the number of stars for $z=0 \mathrm{pc}$ and $H$ is the vertical exponential scaleheight. For $\operatorname{sech}^{2}$ function, $H$ is calculated from $H=1.08504 H_{z}$, where $H_{z}$ is the vertical $\operatorname{sech}^{2}$ scaleheight.

\begin{tabular}{lccccc}
\hline & & \multicolumn{2}{c}{$J_{0} \leq 15.8$} & \multicolumn{2}{c}{$J_{0} \leq 16.5$} \\
Parameter & Subgroup & exp & sech $^{2}$ & exp & sech $^{2}$ \\
\hline$n_{0}$ & DN & $111 \pm 13$ & $83 \pm 8$ & $131 \pm 13$ & $100 \pm 8$ \\
$H(\mathrm{pc})$ & & $128 \pm 20$ & $85 \pm 12$ & $150 \pm 18$ & $99 \pm 12$ \\
$\chi_{\min }^{2}$ & & 1.98 & 4.34 & 3.20 & 2.96 \\
\hline$n_{0}$ & $\mathrm{NL}$ & $63 \pm 7$ & $49 \pm 6$ & $71 \pm 7$ & $57 \pm 5$ \\
$H(\mathrm{pc})$ & & $250 \pm 35$ & $160 \pm 5$ & $278 \pm 30$ & $177 \pm 5$ \\
$\chi_{\min }^{2}$ & & 4.53 & 1.35 & 7.23 & 1.11 \\
\hline$n_{0}$ & $\mathrm{AM}$ & $21 \pm 6$ & $17 \pm 4$ & $29 \pm 5$ & $23 \pm 4$ \\
$H(\mathrm{pc})$ & & $185 \pm 60$ & $119 \pm 35$ & $225 \pm 42$ & $142 \pm 26$ \\
$\chi_{\min }^{2}$ & & 5.17 & 3.29 & 4.64 & 2.21 \\
\hline$n_{0}$ & $\mathrm{NM}$ & $198 \pm 14$ & $151 \pm 14$ & $225 \pm 15$ & $173 \pm 12$ \\
$H(\mathrm{pc})$ & & $154 \pm 15$ & $99 \pm 11$ & $171 \pm 15$ & $110 \pm 10$ \\
$\chi_{\min }^{2}$ & & 3.20 & 12.30 & 2.20 & 8.67 \\
\hline$n_{0}$ & $\mathrm{ALL}$ & $218 \pm 14$ & $167 \pm 13$ & $252 \pm 14$ & $195 \pm 12$ \\
$H(\mathrm{pc})$ & & $158 \pm 14$ & $102 \pm 36$ & $179 \pm 13$ & $115 \pm 9$ \\
$\chi_{\min }^{2}$ & & 2.07 & 15.60 & 1.93 & 6.79 \\
\hline
\end{tabular}

The numbers and scaleheights of polars (magnetic systems-AM Her stars) are estimated separately since the evolution of these systems could be different from the evolution of non-magnetic CVs (Wu \& Wickramasinghe, 1993; Webbink \& Wickramasinghe, 2002).

The number density as a function of $z$ and the scaleheight estimated from the histogram according to the magnitude limit 15.8 are smaller than those from the histogram using the limiting magnitude of 16.5. This must be due to the fact that faint systems are more distant than brighter ones. Figures 5 and 6 show that the exponential function well represents the $z$-histograms of all CVs for both limiting magnitudes. The vertical scaleheights derived from the exponential function for the limiting apparent magnitudes of 15.8 and 16.5 are 158 and $179 \mathrm{pc}$, respectively.

However, according to Table 3, the $z$-histograms for the nova-like stars and polars are better fitted by $\operatorname{sech}^{2}$ functions, while exponential functions give better fits for the other two subgroups. Consequently, the better fitting functions require that the scaleheights of dwarf novae, nova-like stars, polars and non-magnetic systems are 128, 160, 119 and 154 pc, respectively, for the limiting magnitude of 15.8. In the same order, the scaleheights change to 150 , 177, 142 and 171 for the limiting magnitude of 16.5 (see Table 3). 


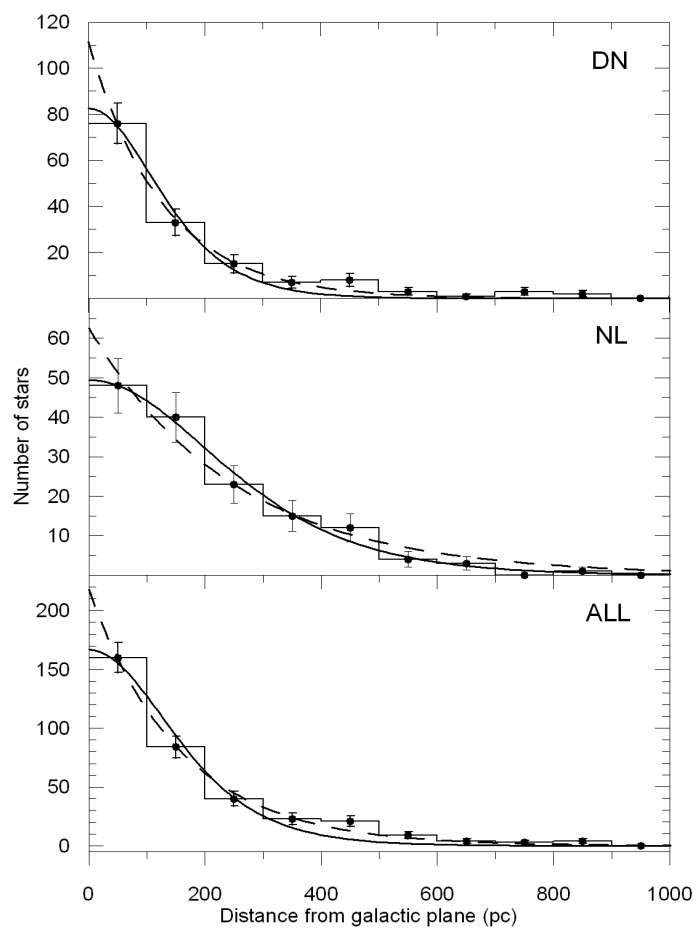

Fig. 5. The $z$-histograms for CVs. The systems are selected according to the limiting apparent magnitude of $J_{0}=15.8$. Here, ALL denotes all CVs in the sample, DN dwarf novae, NL nova-like stars. The dashed line represents the exponential function, while the solid line shows the $\operatorname{sech}^{2}$ function.

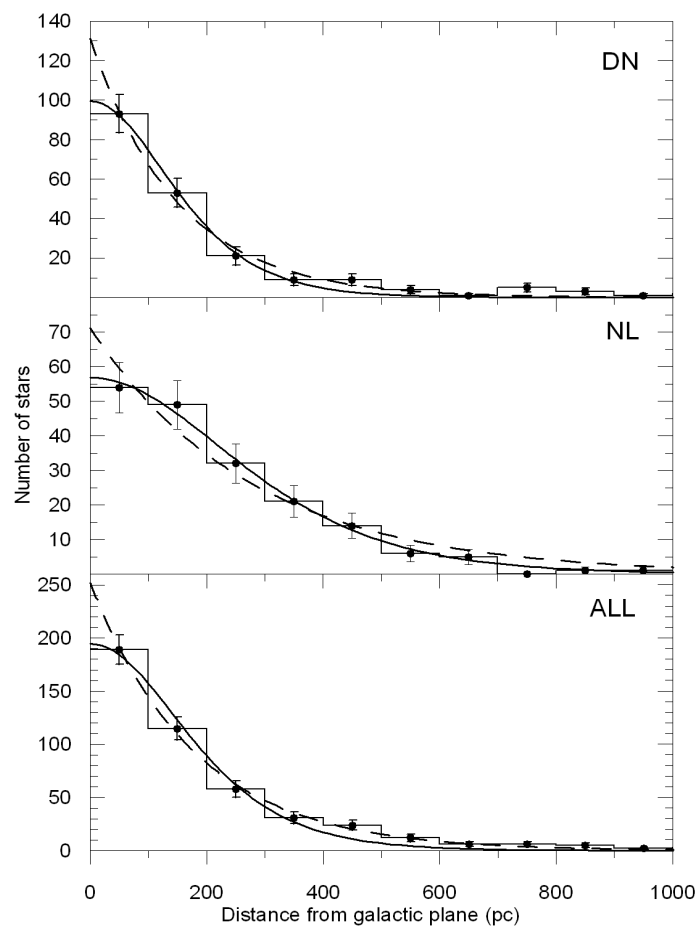

Fig. 6. The $z$-histograms for CVs. The systems are selected according to the limiting apparent magnitude of $J_{0}=16.5$. The denotes and lines are as in Figure 5 . 


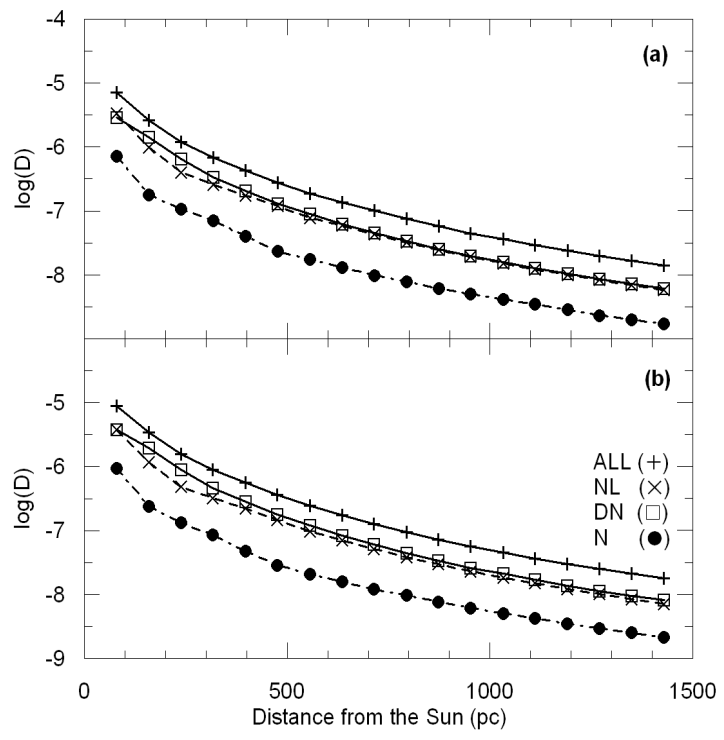

Fig. 7. The logarithmic density functions of CVs. The systems are selected according to the limiting apparent magnitudes of $J_{0}=15.8$ (a) and 16.5 (b). The denotes are as in Figure 5.

Table 4

The local space densities of CVs. The results are given for the limiting magnitudes of $J_{0}=15.8$ and 16.5 .

\begin{tabular}{lcc}
\hline & $J_{0} \leq 15.8$ & $J_{0} \leq 16.5$ \\
Type & $D_{0}\left(\mathrm{pc}^{-3}\right)$ & $D_{0}\left(\mathrm{pc}^{-3}\right)$ \\
\hline Dwarf novae & $6.3( \pm 0.6) \times 10^{-6}$ & $7.9( \pm 0.9) \times 10^{-6}$ \\
Nova-like stars & $2.5( \pm 0.3) \times 10^{-5}$ & $2.8( \pm 0.4) \times 10^{-5}$ \\
Novae & $4.3( \pm 1.2) \times 10^{-6}$ & $5.5( \pm 1.4) \times 10^{-6}$ \\
All systems & $2.9( \pm 0.1) \times 10^{-5}$ & $3.2( \pm 0.1) \times 10^{-5}$ \\
\hline
\end{tabular}

Finally, the local space densities are derived by dividing the cumulative number of stars in consecutive distances $(d)$ from the Sun to the corresponding spherical volumes. The logarithmic density functions of dwarf novae, nova-like stars, novae and all stars in the sample are shown in Figure 7. The local space densities are summarized in Table 4, which shows that our choice of limiting magnitude does not strongly affect the space densities. Moreover, it seems that the space density of novae is smaller than those of dwarf novae and nova-like stars for a factor of $\sim 1.5$ and $\sim 5.8$, respectively.

\subsection{Luminosity function of $C V s$}

The luminosity function is defined as the space density of stars in a certain absolute magnitude interval. The logarithmic luminosity functions of CVs ac- 
Table 5

The logarithmic luminosity functions of CVs in our sample with the limiting apparent magnitude of $J_{0}=15.8 . N$ is the number of stars in the $M_{J 1}-M_{J 2}$ absolute magnitude interval and $\phi$ the logarithmic luminosity function. Distance is in pc, volume $\mathrm{pc}^{3}$.

\begin{tabular}{ccccccccccc}
\hline & & \multicolumn{4}{c}{ Nova-like stars } & \multicolumn{2}{c}{ Dwarf novae } & \multicolumn{2}{c}{ Novae } & \multicolumn{2}{c}{ All systems } \\
$M_{J 1}-M_{J 2}$ & $d_{1}-d_{2}$ & $\Delta V$ & $\mathrm{~N}$ & $\phi$ & $\mathrm{N}$ & $\phi$ & $\mathrm{N}$ & $\phi$ & $\mathrm{N}$ & $\phi$ \\
\hline$(2.5,3.5]$ & $398-3631$ & $2.00(11)$ & 12 & $-10.22 \pm 0.11$ & 5 & $-10.60 \pm 0.16$ & 4 & $-10.70 \pm 0.18$ & 22 & $-9.96 \pm 0.08$ \\
$(3.5,4.5]$ & $251-2291$ & $5.03(10)$ & 13 & $-9.59 \pm 0.11$ & 19 & $-9.42 \pm 0.09$ & 7 & $-9.86 \pm 0.14$ & 39 & $-9.11 \pm 0.06$ \\
$(4.5,5.5]$ & $158-1445$ & $1.26(10)$ & 43 & $-8.47 \pm 0.06$ & 17 & $-8.87 \pm 0.09$ & 11 & $-9.06 \pm 0.11$ & 72 & $-8.24 \pm 0.05$ \\
$(5.5,6.5]$ & $100-912$ & $3.17(9)$ & 27 & $-8.07 \pm 0.08$ & 29 & $-8.04 \pm 0.07$ & 7 & $-8.66 \pm 0.14$ & 65 & $-7.69 \pm 0.05$ \\
$(6.5,7.5]$ & $63-575$ & $7.95(8)$ & 17 & $-7.67 \pm 0.09$ & 29 & $-7.44 \pm 0.07$ & 8 & $-8.00 \pm 0.13$ & 55 & $-7.16 \pm 0.05$ \\
$(7.5,8.5]$ & $40-363$ & $2.00(8)$ & 9 & $-7.35 \pm 0.12$ & 19 & $-7.02 \pm 0.09$ & 4 & $-7.70 \pm 0.18$ & 33 & $-6.78 \pm 0.07$ \\
$(8.5,9.5]$ & $25-229$ & $5.02(7)$ & 13 & $-6.59 \pm 0.11$ & 14 & $-6.55 \pm 0.10$ & 1 & $-7.70 \pm 0.30$ & 28 & $-6.25 \pm 0.08$ \\
$(9.5,10.5]$ & $16-145$ & $1.28(7)$ & 5 & $-6.41 \pm 0.16$ & 12 & $-6.03 \pm 0.11$ & 1 & $-7.11 \pm 0.30$ & 18 & $-5.85 \pm 0.09$ \\
$(10.5,11.5]$ & $10-91$ & $3.15(6)$ & 2 & $-6.20 \pm 0.23$ & 3 & $-6.02 \pm 0.20$ & 0 & & 6 & $-5.72 \pm 0.15$ \\
\hline
\end{tabular}

cording to the limiting apparent magnitude of $J_{0}=15.8$ are listed in Table 5 and plotted in Figure 8.

In Table $5, d_{1}$ and $d_{2}$ correspond to the distances calculated for the lower and upper limiting apparent magnitudes of $J_{0}=11.0$ and 15.8 , respectively, for an absolute magnitude interval. $\Delta V$ is the shell volume between the $d_{1}$ and $d_{2}$ distances and $\phi=\log N / \Delta V$ is the luminosity function. The luminosity functions are estimated for three types of CVs; dwarf novae, nova-like stars, novae and for the combination of all. Figure 8 indicates that the luminosity function of dwarf novae and nova-like stars are similar, while novae have a rather smaller luminosity function. Note that all of the luminosity functions increase towards fainter magnitudes.

In order to compare the luminosity functions of DA white dwarfs found from the Anglo Australian Telescope Survey (AAT, Boyle, 1989) and Palomar Green Survey (PG, Fleming et al., 1986) with the luminosity function of CVs in this study, we first transformed the $M_{J}$ absolute magnitudes of CVs to Johnson $M_{V}$ absolute magnitudes by using Padova Isochrones (Girardi et al., 2002; Bonatto et al., 2004). In the selection of the isochrones, we have considered the spatial distribution of CVs as located in the old thin disc of the Galaxy, in general. Thus, we have obtained an analytical relation between $M_{J}$ and $M_{V}$ by assuming a mass fraction of metals of $Z=0.008$, a logarithmic surface gravity of $\log g>4$ and a mean age of $t=5.01$ Gyr. The $\chi_{\text {min }}^{2}$ analysis show that the best fits between the luminosity functions of CVs and white dwarfs are obtained by dividing the luminosity functions of white dwarfs to 200 and 400 for the PG and AAT surveys, respectively. Comparison of luminosity functions are demonstrated in Figure 9. We conclude that the present luminosity function of CVs in this study for $M_{V}>10^{m}$ is reasonably compatible with the luminosity function of DA white dwarfs obtained from AAT 


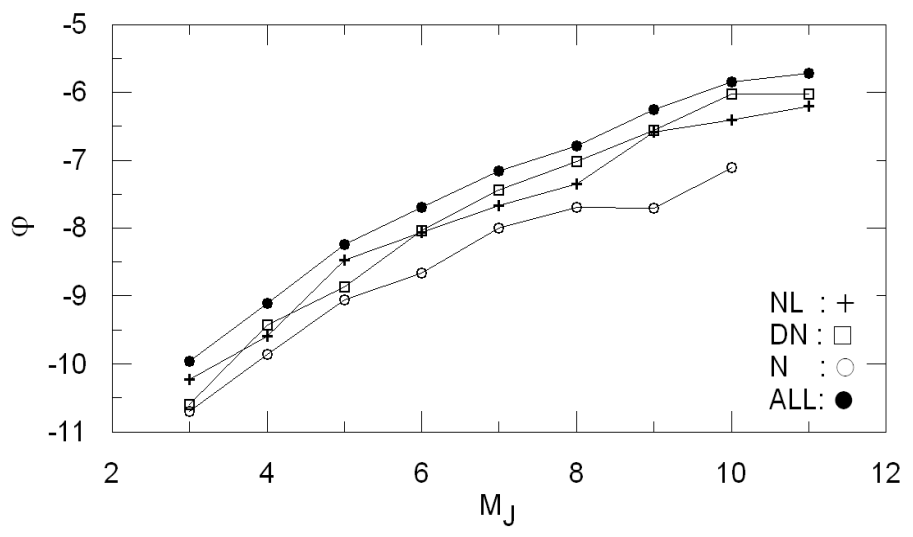

Fig. 8. The logarithmic luminosity functions of CVs in our sample with the limiting apparent magnitude of $J_{0}=15.8$. The denotes are as in Figure 5.

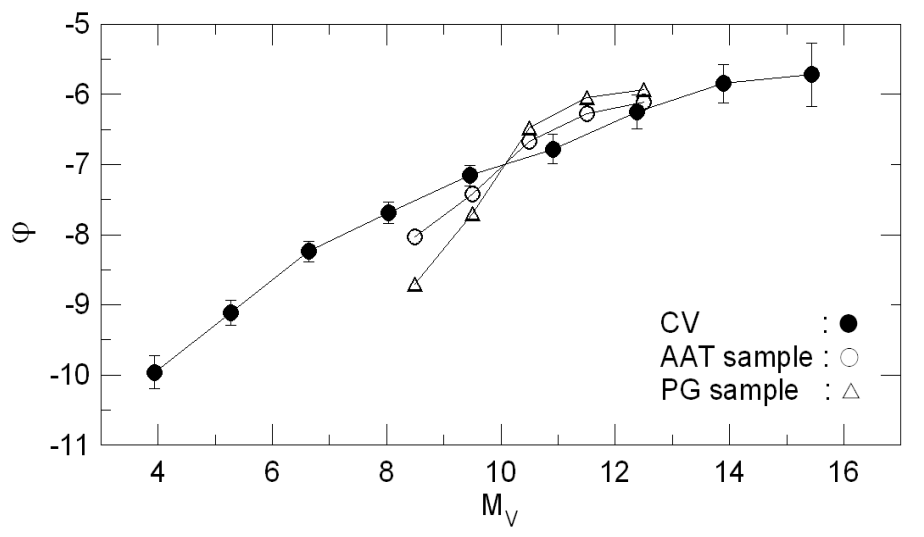

Fig. 9. Comparison of the luminosity functions of DA white dwarfs derived from the AAT (Boyle, 1989) and PG surveys (Fleming et al., 1986) with the luminosity function of CVs in this study.

survey.

\section{Conclusions}

The spatial distribution, galactic model parameters and luminosity function of CVs for the solar neighbourhood have been derived. In computing the CV distances, a new method suggested by Ak et al. (2007), which uses orbital periods and intrinsic $2 M A S S$ infrared colours, was adopted. This new method advantageously allowed us to collect a fairly homogeneous sample of CVs regarding to the distances. 


\subsection{Spatial distribution of $C V s$}

According to Figure 3 and Table 2, most CVs appear to be closer than $1 \mathrm{kpc}$ to the Sun, while they are located within the galactic disc with $z$ distances not higher than $0.5 \mathrm{kpc}$ from the galactic plane, in general. Novae are strongly concentrated towards the galactic plane. Consequently, it might be concluded that because novae share the same galactic regions with the young-thin disc population, they could as well be members of the young population. Such a result would be in agreement with that of Duerbeck (1984) who suggested that most novae come from fairly young stellar progenitors (see also, Hatano et al., 1997; Della Valle \& Livio, 1998). As for the spatial distribution of other CV types, the homogeneous spatial distribution of dwarf novae and nova-like stars implies that these systems are formed both in young and old populations in the galactic disc (see Figure 3). According to van Paradijs et al. (1996), the velocity distribution of CVs indicates that they are an old disc population, with a mix of ages up to 10 Gyr. Clarification of this point, however, requires further work on the space velocity dispersions of $\mathrm{CVs}_{\mathrm{s}}$ and their subgroups. Our forthcoming work will be dedicated to studying the space velocity fields and kinematical ages of CVs.

\subsection{Galactic model parameters of $C V s$}

The $z$-histograms for the nova-like stars and polars are well represented by the $\operatorname{sech}^{2}$ functions, while the $z$-histograms of dwarf novae are well fitted by an exponential function. In observational and theoretical population studies of CVs, usually an exponential distribution function has been assumed. However, it should be noted that the observed vertical distribution concerning the stars of all kinds in the Galaxy is flat, and better-approximated by a sech ${ }^{2}$ function (Bilir et al., 2006).

It has been found in this study that the exponential vertical scaleheights of CVs are 158 and 179 pc for the limiting apparent magnitudes of 15.8 and 16.5, respectively. These values are compatible with the exponential scaleheights in the range of 100-250 pc and 160-230 pc suggested by Patterson (1984) and van Paradijs et al. (1996), respectively. On the other hand, the present data and analysis indicate the vertical scaleheights of nova-like stars and polars are within the ranges $160-177 \mathrm{pc}$ and $119-142 \mathrm{pc}$, respectively. These values are very much comparable with the scaleheight of $\sim 155 \mathrm{pc}$ determined by Thomas \& Beuermann (1998) for the polars. Finally, we have found that the exponential vertical scaleheight of dwarf novae is 128 and $150 \mathrm{pc}$ for the two limiting apparent magnitudes. The first of these values is in agreement with the scaleheight of $119 \pm 9 \mathrm{pc}$ given by Duerbeck (1984). 
Schwope et al. (2002) claimed that 10 to 100 undetected CVs should be hiding in the local vicinity $(50 \mathrm{pc})$ of the Sun. According to our analysis, if the $z$ distribution of CVs is modeled by an exponential function, there should be about 50 undetected systems in the solar neighbourhood. However, if the $z$ distribution of CVs is modeled by a $\operatorname{sech}^{2}$ function, as indicated in Figures 5 and 6, we would only miss at most $10 \mathrm{CVs}$ in the solar neighbourhood. Consequently, we could claim that the discrepancies between the theoretical and observational population studies of CVs would almost be removed if the $\operatorname{sech}^{2}$ density function is accepted in the theoretical population studies.

\subsection{Space density of $C V s$}

The local space densities calculated according to the limiting apparent magnitude of 15.8 define a lower limit. However, the local densities do not change considerably when we use the limiting apparent magnitude of 16.5, which means that our estimates cannot deviate very much from the true space densities.

According to present sample and its analysis the local space density of CVs is $2.9( \pm 0.1) \times 10^{-5} \mathrm{pc}^{-3}$. However, former observational population studies of CVs gave predictions of space densities from $10^{-6}$ up to $3 \times 10^{-5}$ $\mathrm{pc}^{-3}$ (Duerbeck, 1984; Patterson, 1998; Hertz et al., 1990; Schwope et al., 2002; Grindlay et al., 2005). On the other hand, the theoretical population studies of CVs predicted local space densities between $10^{-5}$ and $10^{-4} \mathrm{pc}^{-3}$ (Ritter \& Burkert, 1986; de Kool, 1992; Politano, 1996). Furthermore, Shara et al. (1986) argued that surveys for CVs are very incomplete, and that the space density of CVs could well be $10^{-4} \mathrm{pc}^{-3}$. However, Kolb (2001) claimed that the space density should be smaller than $10^{-4} \mathrm{pc}^{-3}$ if the initial mass ratio distribution is more sharply peaked to unity. Thus, the intrinsic space density of CVs could well be close to the observational value derived here $\sim 3 \times 10^{-5} \mathrm{pc}^{-3}$. The local space density of CVs predicted from the present sample confirms Kolb's (2001) theoretical work.

The local space density of dwarf novae was found to be $6.3( \pm 0.6) \times 10^{-6}$ $\mathrm{pc}^{-3}$ (Table 4). This value is in agreement with the space density of $6 \times 10^{-6}$ $\mathrm{pc}^{-3}$ found by Ringwald (1993), who obtained a higher space density than the observational results of Duerbeck (1984) and Downes (1986). For the nova-like stars in the present CV sample, we have derived a space density of $2.6( \pm 0.3) \times$ $10^{-5} \mathrm{pc}^{-3}$. This value is about $\sim 60$ times higher than the space density of nova-like stars estimated by Downes (1986). On the other hand, space density of novae in the present CV sample is $4.3( \pm 1.2) \times 10^{-6} \mathrm{pc}^{-3}$. This could be interpreted as that the space density derived in this study is in agreement with $2.2-4.4 \times 10^{-6} \mathrm{pc}^{-3}$ found by Patterson (1984), which is considerably 
higher than the space densities of $1.4 \times 10^{-7}$ and $7 \times 10^{-7} \mathrm{pc}^{-3}$ estimated by Downes (1986) and Della Valle \& Duerbeck (1993), respectively. One of the most important challenges of predicting the space densities is the prediction of the true distances. Relying on a homogenized distance prediction, we could claim the space densities found in this study are more reliable than in the previous studies.

\subsection{Luminosity function of $C V s$}

The luminosity function of CVs increases towards fainter absolute magnitudes. Similar trends are clear for all types of CVs studied in the present sample. The luminosity function of CVs showing such a trend was first examined by Ringwald (1993). This trend clearly implies an increase in the number of shortperiod systems. This is a result which is roughly consistent with the theoretical prediction of Kolb (1993), who suggested that $\sim 99$ per cent of all CVs should be intrinsically below the period gap. The theory predicts that most of CVs should be intrinsically faint (Howell, Rappaport \& Politano, 1997).

We have compared the luminosity function of CVs in the present study with the luminosity functions of DA white dwarfs found from the AAT Survey by Boyle (1989) and PG Survey by Fleming et al. (1986) (see also, Hu et al., 2007). Our analysis show that the best fit between the luminosity functions of CVs and DA white dwarfs can be obtained by dividing the luminosity function of DA white dwarfs by 400 for the AAT surveys. We conclude thereby that one CV is formed for every 400 DA white dwarfs in the solar neighbourhood. However, it should be noted that it is not clearly known if CV white dwarfs share the narrow mass distribution of isolated DA degenerates (Sion, 1999).

\section{Acknowledgments}

We thank the anonymous referee for a thorough report and useful comments that helped improving an early version of the paper. Part of this work was supported by the Research Fund of the University of Istanbul, Project Numbers: BYP-723/24062005 and BYP-1379. This research has made use of the SIMBAD database, operated at CDS, Strasbourg, France. This publication makes use of data products from the Two Micron All Sky Survey, which is a joint project of the University of Massachusetts and the Infrared Processing and Analysis Center/California Institute of Technology, funded by the National Aeronautics and Space Administration and the National Science Foundation. This research has made use of the NASA/IPAC Extragalactic Database (NED) which is operated by the Jet Propulsion Laboratory, California Institute of 
Technology, under contract with the National Aeronautics and Space Administration. This research has made use of NASA's Astrophysics Data System.

\section{References}

Ak, T., Bilir, S., Ak, S., Retter, A., 2007. NewA 12, 446

Araujo-Betancor, S.,Gänsicke, B.T., Long, K.S., Beuermann, K., de Martino, D., Sion, E.M., Szkody, P., 2005. ApJ 622, 589

Aungwerojwit, A., Gänsicke, B.T., Rodriguez-Gil, P., Hagen, H.J., Harlaftis, E.T., Papadimitriou, C., Lehto, H., Araujo-Betancor, S., Heber, U., Fried, R.E., Engels, D., Katajainen, S., 2005. A\&A 443, 995

Bahcall, J.N., Soneira, R.M., 1980. ApJS 44, 73

Bilir, S., Karaali, S., Ak, S., Yaz, E., Hamzaoḡlu, E., 2006. NewA 12, 234

Bilir, S., Güver, T., Aslan, M., 2006. AN 327, 693

Bonatto, Ch., Bica, E., Girardi, L., 2004. A\&A 415, 571

Boyle, B.J., 1989. MNRAS 240, 549

Bruch, A., Engel, A., 1994. A\&AS 104, 79

Cutri, R.M. et al., 2003. VizieR On-Line Data Catalog: II/246, Originally published in : University of Massachusetts and Infrared Processing and Analysis Center

Della Valle, M., Duerbeck, H.W., 1993. A\&A 271, 175

Della Valle, M., Livio, M., 1996. ApJ 457, L77

Della Valle, M., Livio, M., 1998. ApJ 506, 818

de Kool, M., 1992. A\&A 261, 188

Downes, R.A., 1986. ApJ 307, 170

Downes, R.A., Webbink, R.F., Shara, M.M., Ritter, H., Kolb, U., Duerbeck, H.W., 2001. PASP 113, 764

Duerbeck, H.W., 1984. Ap\&SS 99, 363

Fiorucci, M., Munari, U., 2003. A\&A 401, 781

Fleming, T.A., Liebert, J., Green, R.F., 1986. ApJ 308, 176

Gänsicke, B.T., 2005. in The Astrophysics of Cataclysmic Variables and Related Objects, ASP Conf. Ser., Vol.330, J.-M. Hameury, J.-P. Lasota eds., 3

Girardi, L., Bertelli,G., Bressan, A., Chiosi, C., Groenewegen, M.A.T., Marigo, P., Salasnich, B., Weiss, A., 2002. A\&A 391, 195

Grindlay, J.E., Hong, J., Zhao, P., Laycock, S., van den Berg, M., Koenig, X., Schlegel, E.M., Cohn, H.N., Lugger, P.M., Rogel, A.B., 2005. ApJ 635, 920

Hameury, J.M, King, A.R., Lasota, J.P., Ritter, H., 1988. MNRAS 231, 535

Hatano, K., Branch, D., Fisher, A., Starrfield, S., 1997. MNRAS 290, 113

Hellier, C., 2001. Cataclysmic Variable Stars, How and why they vary, Springer-Praxis Books in astronomy and space sciences, Cornwall, UK

Hertz, P., Bailyn, C. D., Grindlay, J. E., Garcia, M. R., Cohn, H., Lugger, P. M., 1990. ApJ 364, 251 
Howell, S.B., Rappaport, S., Politano, M., 1997. MNRAS 287, 929

Howell, S.B., Nelson, L.A., Rappaport, S., 2001. ApJ 550, 897

Hu, Q., Wu, C., Wu, X.-B., 2007. astro-ph/0701197v2

Jurić, M., et al., 2005. astro-ph/0510520

Knigge, C., 2006. MNRAS 373, 484

Kolb, U., 1993. A\&A 271, 149

Kolb, U., 2001. in The influence of binaries on stellar population studies, Dordrecht: Kluwer Academic Publishers, Astrophysics and Space Science Library, Vol. 264, 321

Mendez, R.A., van Altena, W.F., 1998. A\&A 330, 910

Patterson, J., 1984. ApJS 54, 443

Patterson, J., 1998. PASP 110, 1132

Patterson, J., 2001. PASP 113, 736

Politano, M., 1996. ApJ 465, 338

Press, W.H., Teukolsky, S.A., Vetterling, W.T., Flannery, B.P., 1997. Numerical Recipes in Fortran 77 - The Art of Scientific Computing, Second Edition, ISBN 0-521-43064-X, Cambridge Univ. Press., 688

Pretorius, M.L., Knigge, C., Kolb, U., 2007. MNRAS 374, 1495

Ringwald, F.A., 1993. PASP 105, 805

Ritter, H., Burkert, A., 1986. A\&A 158, 161

Ritter, H., Kolb, U., 2003. A\&A 404, 301

Schlegel, D.J., Finkbeiner, D.P., Davis, M., 1998. ApJ 500, 525

Schwope, A.D., Brunner, H., Buckley, D., Greiner, J., van den Heyden, K., Neizvestny, S., Potter, S., Schwarz, R., 2002. A\&A 396, 895

Shara, M.M., Livio, M., Moffat, A.F.J., Orio, M., 1986. ApJ 311, 163

Sion, E.M., 1999. PASP 111, 532

Smith, D.A., Dhillon, V.S., 1998. MNRAS 301, 767

Spruit, H.C., Ritter, H., 1983. A\&A 124, 267

Skrutskie, M.F., Cutri, R.M., Stiening, R., Weinberg, M.D., Schneider, S., et al., 2006. AJ 131, 1163

Thomas, H.-C., Beuermann, K., 1998. in The Local Bubble and Beyond, IAU Colloq. No.166, D. Breitschwerdt, M.J. Freyberg, J. Truemper eds., 247

van Paradijs, J., Augusteijn, T., Stehle, R., 1996. A\&A 312, 93

Warner, B., 1974. MNRAS 167, 61 UV and X-Ray Spectroscopy of Astrophysical and Laboratory Plasmas, ed. P.K. Carroll (Dublin: Univ. Dublin, Physics Dept.), 85

Warner, B., 1995. Cataclysmic Variable Stars, Cambridge Univ. Press, Cambridge

Webbink, R.F., Wickramasinghe, D.T., 2002. MNRAS 335, 1

Willems, B., Kolb, U., Sandquist, E.L., Taam, R.E., Dubus, G., 2005. ApJ 635,1263

Willems, B., Taam, R.E., Kolb, U., Dubus, G., Sandquist, E.L., 2006. astroph/0611569

Wu, K., Wickramasinghe, D.T., 1993. MNRAS 265, 115 